\title{
Neutrino emission from superfluid neutron-star cores: Various types of neutron pairing
}

\author{
M. E. Gusakov` \\ Ioffe Physical Technical Institute, Politekhnicheskaya 26, 194021 St. Petersburg, Russia \\ Received 12 March 2002 / Accepted 16 April 2002

\begin{abstract}
We calculate and provide analytic fits of the factors which describe the reduction of the neutrino emissivity of modified Urca and nucleon-nucleon bremsstrahlung processes by superfluidity of neutrons and protons in neutron-star cores. We consider ${ }^{1} \mathrm{~S}_{0}$ pairing of protons and either ${ }^{1} \mathrm{~S}_{0}$ or ${ }^{3} \mathrm{P}_{2}$ pairing of neutrons. We analyze two types of ${ }^{3} \mathrm{P}_{2}$ pairing: the familiar pairing with zero projection of the total angular momentum of neutron pairs onto quantization axis, $m_{\mathrm{J}}=0$; and the pairing with $\left|m_{\mathrm{J}}\right|=2$ which leads to the gap with nodes at the neutron Fermi surface. Combining the new data with those available in the literature we fully describe neutrino emission by nucleons from neutron star cores to be used in simulations of cooling of superfluid neutron stars.
\end{abstract}

Key words. stars: neutron - dense matter

\section{Introduction}

It is well-known (e.g., Yakovlev et al. 1999, 2001) that cooling of neutron stars depends on the properties of matter in the neutron star cores. In spite of great progress in understanding the neutron-star physics, many properties of this matter are still known with large uncertainty. A comparison of the theoretical cooling models with observations of thermal emission from isolated neutron stars gives a potentially powerful method to explore the internal structure of neutron stars. For a successful modeling of the cooling one needs reliable values of neutrino emissivity in different neutrino reactions.

In this paper we consider the matter of neutron star cores (at densities $\rho \gtrsim 1.5 \times 10^{14} \mathrm{~g} \mathrm{~cm}^{-3}$ ) composed of neutrons (n), protons (p), and electrons (e). It is generally agreed the neutrons and protons can be in superfluid state (as reviewed, e.g., by Lombardo \& Schulze 2001). Superfluidity affects the neutrino emission and thus the cooling of neutron stars. According to numerous microscopic calculations, the proton pairing occurs in the singlet $\left({ }^{1} \mathrm{~S}_{0}\right)$ state of proton pairs. Following Yakovlev et al. (1999) we will call this pairing as pairing A. The neutron pairing occurs either in the ${ }^{1} \mathrm{~S}_{0}$ state or in the triplet state $\left({ }^{3} \mathrm{P}_{2}\right)$. Neutron pairing A takes place in the matter of subnuclear density $\left(\rho \lesssim \rho_{0}\right.$, where $\rho_{0}=2.8 \times 10^{14} \mathrm{~g} \mathrm{~cm}^{-3}$ is the saturated nuclear matter density), while the ${ }^{3} \mathrm{P}_{2}$ pairing is efficient at higher $\rho$. We consider the ${ }^{3} \mathrm{P}_{2}$ pairing of two types denoted as $\mathrm{B}$ and C. Pairing $\mathrm{B}$ occurs in a state of a neutron pair with zero projection of the total angular momentum on the quantization axis, $m_{\mathrm{J}}=0$. This

* e-mail: gusakov@astro.ioffe.rssi.ru pairing has been studied in the majority of papers devoted to ${ }^{3} \mathrm{P}_{2}$ pairing of neutrons. Pairing $\mathrm{C}$ occurs in a state with $\left|m_{\mathrm{J}}\right|=2$. It has been the subject of some studies (as reviewed, e.g., by Yakovlev et al. 1999). The actual type of neutron pairing $(\mathrm{A}, \mathrm{B}$, or $\mathrm{C})$ corresponds to the state with minimum free energy. Pairing $\mathrm{C}$ seems to be less realistic than B but cannot be completely ruled out by contemporary microscopic theories. For example, Muzikar et al. (1980) showed that it realizes in matter with strong magnetic field $\left(B \gtrsim 10^{16} \mathrm{G}\right)$. Amundsen \& Østgaard (1985) found that the energetically preferable state of the pair can be a superposition of states with different $m_{\mathrm{J}}$. The specific feature of pairing $\mathrm{C}$ is that it leads to superfluid gaps with nodes at the neutron Fermi surface producing qualitatively different effect on neutrino processes than pairing B (or A).

Note that we do not consider another case: ${ }^{3} \mathrm{P}_{2}$ neutron pairing with $\left|m_{\mathrm{J}}\right|=1$. In this case, just as in cases $\mathrm{A}$ and $\mathrm{B}$, the superfluid gap does not have any nodes at the Fermi surface (e.g., Amundsen \& Østgaard 1985). Therefore, we expect that the results will be similar to those for pairing $\mathrm{B}$ or $\mathrm{A}$. On the other hand, the consideration of the $\left|m_{\mathrm{J}}\right|=1$ pairing is technically much more complicated since the superfluid gap depends not only on the polar angle $\vartheta$ of neutron momentum at the Fermi sphere (see below) but also on the azimuthal angle $\varphi$.

Let us remind five main neutrino generation mechanisms in the neutron-star cores.

(1) Direct Urca process is the most powerful neutrino process. It consists of two successive reactions

$\mathrm{n} \rightarrow \mathrm{p}+\mathrm{e}+\bar{\nu}_{\mathrm{e}}, \quad \mathrm{p}+\mathrm{e} \rightarrow \mathrm{n}+\nu_{\mathrm{e}}$ 
where $\nu_{\mathrm{e}}$ and $\bar{\nu}_{\mathrm{e}}$ are electron neutrino and antineutrino. It is allowed (Lattimer et al. 1991) only in matter of sufficiently high density (typically, a few times of $\rho_{0}$ ) for model equations of state with high symmetry energy (rather high fraction of protons). The reduction of direct Urca process by proton superfluidity A and neutron superfluidity (A, B, or C) was analyzed by Levenfish \& Yakovlev (1994).

(2) Modified Urca process consists of two branches. Two successive reactions (direct and inverse)

$\mathrm{n}+\mathrm{n} \rightarrow \mathrm{p}+\mathrm{n}+\mathrm{e}+\bar{\nu}_{\mathrm{e}}, \quad \mathrm{p}+\mathrm{n}+\mathrm{e} \rightarrow \mathrm{n}+\mathrm{n}+\nu_{\mathrm{e}}$

form the neutron branch. Two similar reactions

$\mathrm{n}+\mathrm{p} \rightarrow \mathrm{p}+\mathrm{p}+\mathrm{e}+\bar{\nu}_{\mathrm{e}}, \quad \mathrm{p}+\mathrm{p}+\mathrm{e} \rightarrow \mathrm{n}+\mathrm{p}+\nu_{\mathrm{e}}$

form the proton branch of the process. The process is the most powerful neutrino mechanism in non-superfluid neutron-star cores where the direct Urca process is forbidden. It (or at least its neutron branch) is open in the entire stellar core.

The neutrino emissivity of this process in nonsuperfluid matter was considered by a number of authors (references can be found in Yakovlev et al. 1999), particularly, by Bahcall \& Wolf (1965), Friman \& Maxwell (1979), and Yakovlev \& Levenfish (1995). The latter authors studied the reduction of the process either by proton superfluidity A, or by neutron superfluidity (A or B). Levenfish \& Yakovlev (1996) suggested a simple approximate method to account for the combined effect of the neutron and proton superfluidities. It is based on the similarity relations of the factors which describe the superfluid reduction of the direct and modified Urca processes. These results were used in simulations of the neutron star cooling (as reviewed by Yakovlev et al. 1999, 2001). We present a more accurate calculation of the reduction of the modified Urca process by combined action of proton superfluidity A and neutron superfluidity (A, B, or C).

(3) The neutrino-pair bremsstrahlung at nucleonnucleon scattering can be of three types:

$\mathrm{n}+\mathrm{n} \rightarrow \mathrm{n}+\mathrm{n}+\nu+\bar{\nu}$
$\mathrm{n}+\mathrm{p} \rightarrow \mathrm{n}+\mathrm{p}+\nu+\bar{\nu}$
$\mathrm{p}+\mathrm{p} \rightarrow \mathrm{p}+\mathrm{p}+\nu+\bar{\nu}$.

Here, $\nu$ and $\bar{\nu}$ stand for neutrinos of any flavor. In a normal (non-superfluid) matter the bremsstrahlung processes are weaker than the modified Urca process (e.g., Yakovlev et al. 1999). However they may be more important in superfluid matter. If proton superfluidity is of type A, and neutron superfluidity is of type A or B then superfluid reduction of the processes can be described by the formulae presented by Yakovlev et al. (1999). In this paper we develop analogous description for neutron superfluidity C.

(4) Neutrino emission due to Cooper pairing of nucleons $(\mathrm{N}=\mathrm{n}$ or $\mathrm{p}$ ) actually consists of neutrino-pair (any flavor) emission

$\mathrm{N} \rightarrow \mathrm{N}+\nu+\bar{\nu}$ by a nucleon whose dispersion relation contains an energy gap. The process was proposed by Flowers et al. (1976) for neutron superfluidity of type A. The extension to the neutron superfluidity B and C was done by Yakovlev et al. (1999). The case of proton superfluidity A is described, e.g., by Yakovlev et al. (1999, 2001). In the absence of superfluidity, the reaction is forbidden by energy-momentum conservation.

(5) Neutrino-pair bremsstrahlung at electron-electron scattering (Kaminker \& Haensel 1999),

$\mathrm{e}+\mathrm{e} \rightarrow \mathrm{e}+\mathrm{e}+\nu+\bar{\nu}$

is much weaker than other processes in non-superfluid matter. However, it is almost independent of superfluidity and may be the leading mechanism in superfluid matter.

The present paper is organized as follows. In Sect. 2 we present general equations for modified Urca process and analyze the reduction factors. In Sect. 3 we consider the reduction factors of nucleon-nucleon bremsstrahlung processes. In Sect. 4 we study the efficiency of various neutrino processes in the cores of neutron stars for different superfluidity types. Analytic fits of the reduction factors of the modified Urca process are given in Appendix.

\section{Modified Urca process}

\subsection{General equations}

As discussed, e.g., by Bahcall \& Wolf (1965) and Friman \& Maxwell (1979), the general expression for the neutrino emissivity of modified Urca process can be written as $(\hbar=$ $\left.c=k_{\mathrm{B}}=1\right)$ :

$$
\begin{aligned}
Q= & 2 \int\left[\prod_{j=1}^{4} \frac{\mathrm{d}^{3} p_{j}}{(2 \pi)^{3}}\right] \frac{\mathrm{d}^{3} p_{\mathrm{e}}}{2 \varepsilon_{\mathrm{e}}(2 \pi)^{3}} \frac{\mathrm{d}^{3} p_{\nu}}{2 \varepsilon_{\nu}(2 \pi)^{3}} \varepsilon_{\nu} \\
& \times(2 \pi)^{4} \delta\left(E_{f}-E_{i}\right) \delta\left(\boldsymbol{P}_{f}-\boldsymbol{P}_{i}\right) \frac{\mathcal{L}}{2} \sum_{\text {spins }}|M|^{2},
\end{aligned}
$$

where $\boldsymbol{p}_{j}$ is a nucleon momentum $(j=1,2,3,4) ; \boldsymbol{p}_{\mathrm{e}}$ and $\varepsilon_{\mathrm{e}}$ are, respectively, the momentum and energy of an electron; and $\boldsymbol{p}_{\nu}$ and $\varepsilon_{\nu}$ are the momentum and energy of a neutrino. The delta function $\delta\left(E_{f}-E_{i}\right)$ describes energy conservation, while $\delta\left(\boldsymbol{P}_{f}-\boldsymbol{P}_{i}\right)$ describes momentum conservation. The indices $i$ and $f$ refer to the initial and final particle states. Furthermore, $\mathcal{L}$ means the product of Fermi-Dirac functions or corresponding blocking factors of the nucleons and the electron; $|M|^{2}$ is the squared matrix element. Summation is carried over all particle spins. The factor 2 in the denominator before the summation sign is introduced to avoid double counting of the same reactions involving identical particles. The overall factor 2 doubles the emissivity of elementary (direct or inverse) reaction of the process assuming beta-equilibrium. Since neutrons, protons and electrons in neutron-star cores are strongly degenerate, one can use the phase-space decomposition 
(e.g., Friman \& Maxwell 1979) which yields:

$$
\begin{aligned}
Q & =\frac{1}{4(2 \pi)^{14}} T^{8} A I \prod_{j=1}^{5} p_{\mathrm{F} j} m_{j}^{*} \sum_{\text {spins }}|M|^{2} \\
A & =4 \pi\left[\prod_{j=1}^{5} \int \mathrm{d} \Omega_{j}\right] \delta\left(\sum_{j=1}^{5} \boldsymbol{p}_{j}\right) \\
I & =\int_{0}^{\infty} \mathrm{d} x_{\nu} x_{\nu}^{3}\left[\prod_{j=1}^{5} \int_{-\infty}^{+\infty} \mathrm{d} x_{j} f_{j}\right] \delta\left(\sum_{j=1}^{5} x_{j}-x_{\nu}\right)
\end{aligned}
$$

The factor $A$ contains integrations over orientation of particle momenta ( $j=5$ refers to an electron); $\mathrm{d} \Omega_{j}$ is a solid angle element in the direction of $\boldsymbol{p}_{j}$. All lengths of the momenta $\boldsymbol{p}_{j}$ with $j \leq 5$ can be set equal to the appropriate Fermi momenta $p_{\mathrm{F}}$. A typical neutrino momentum $p_{\nu}$ is determined by the temperature $T, p_{\nu} \sim T \ll p_{\mathrm{F}}$. Thus, we neglect $p_{\nu}$ in the momentum-conserving delta function and integrate over orientations of $\boldsymbol{p}_{\nu}$ in $A$ (which gives a factor of $4 \pi$ ). The factor $I$ given by Eq. (12) contains the integrals over the dimensionless neutrino energy $x_{\nu}=p_{\nu} / T=\varepsilon_{\nu} / T$ and the dimensionless energies of other particles $x_{j}=v_{\mathrm{F} j}\left(p-p_{\mathrm{F} j}\right) / T ; \quad f_{j}=\left[\exp \left(x_{j}\right)+1\right]^{-1}$. Finally, Eq. (10) contains the products of the densities of state of the particle species $1 \leq j \leq 5, m_{j}^{*}$ being the effective mass at the Fermi surface. For non-relativistic nucleons (which we consider here), $m_{j}^{*}$ is mainly determined by in-medium effects. For the electrons $(j=5), m^{*}=\mu$, where $\mu$ is the electron chemical potential. In the absence of superfluidity Eq. (12) gives $I=I_{0}=11513 \pi^{8} / 120960$. For the neutron branch (2) of the process, Eq. (11) yields (e.g., Shapiro \& Teukolsky 1983):

$A_{0}^{\mathrm{n}}=\frac{2 \pi(4 \pi)^{4}}{p_{\mathrm{Fn}}^{3}}$.

This result is valid as long as $p_{\mathrm{Fn}}>p_{\mathrm{Fp}}+p_{\mathrm{Fe}}$. Otherwise $A_{0}^{\mathrm{n}}$ is given by Eq. (13) in Yakovlev \& Levenfish (1995) but in that case the modified Urca process becomes insignificant because the direct Urca process dominates.

For the proton branch (3) at $p_{\mathrm{Fn}} \geq 3 p_{\mathrm{Fp}}-p_{\mathrm{Fe}}$ one has

$A_{0}^{\mathrm{p}}=\frac{2(2 \pi)^{5}}{p_{\mathrm{Fn}} p_{\mathrm{Fp}}^{3} p_{\mathrm{Fe}}}\left(p_{\mathrm{Fe}}+3 p_{\mathrm{Fp}}-p_{\mathrm{Fn}}\right)^{2} \Theta$

while at $p_{\mathrm{Fn}}<3 p_{\mathrm{Fp}}-p_{\mathrm{Fe}} \quad\left(\right.$ and $\left.3 p_{\mathrm{Fp}} \geq p_{\mathrm{Fe}}\right)$

$A_{0}^{\mathrm{p}}=\frac{2^{3}(2 \pi)^{5}}{p_{\mathrm{Fp}}^{2}}\left(\frac{3}{p_{\mathrm{Fn}}}-\frac{1}{p_{\mathrm{Fp}}}\right) \Theta$,

where $\Theta=1$ if the proton branch is allowed by momentum conservation $\left(p_{\mathrm{Fn}} \leq 3 p_{\mathrm{Fp}}+p_{\mathrm{Fe}}\right)$, and $\Theta=0$ otherwise. Notice that Eq. (15) can be useful if dense matter contains other particles but n, p, and e (e.g., muons).

The difference of Eqs. (13) and (14) or (15) is the consequence of the fact that $p_{\mathrm{Fn}}$ is significantly larger than $p_{\mathrm{Fp}}$ in neutron star matter.
Combining these results one can obtain the neutrino emissivities $Q_{0}^{\mathrm{n}}$ and $Q_{0}^{\mathrm{p}}$ in nonsuperfluid matter. The emissivity $Q_{0}^{\mathrm{n}}$ was calculated by Friman \& Maxwell (1979), using the one-pion-exchange approximation for calculating the matrix element, $|M|^{2}$, and $Q_{0}^{\mathrm{p}}$ was calculated by Yakovlev \& Levenfish (1995) using the same technique.

Now consider the modified Urca process in the presence of superfluidity of neutrons and protons. A onset of superfluidity leads to the appearance of an energy gap $\delta$ in the momentum dependence of the particle energy $\varepsilon(\boldsymbol{p})$. Near the Fermi surface $\left(\left|p-p_{\mathrm{F}}\right| \ll p_{\mathrm{F}}\right)$, this dependence can be written as (e.g., Lifshitz \& Pitaevskii 1980)

$\begin{array}{ll}\varepsilon=\mu-\sqrt{\delta^{2}+\eta^{2}} & p<p_{\mathrm{F}}, \\ \varepsilon=\mu+\sqrt{\delta^{2}+\eta^{2}} & p \geq p_{\mathrm{F}} .\end{array}$

Here, $\eta=v_{\mathrm{F}}\left(p-p_{\mathrm{F}}\right), v_{\mathrm{F}}$ and $p_{\mathrm{F}}$ are the nucleon Fermi velocity and Fermi momentum, and $\mu$ is their chemical potential. For the conditions of our interest, $\delta \ll \mu$. Furthermore, $\delta^{2}=\Delta^{2}(T) F(\vartheta)$, where $\Delta(T)$ is the gap amplitude, which determines the temperature dependence of the gap width, while $F(\vartheta)$ is the factor which depends on the angle $\vartheta$ between the quantization axis and the particle momentum. The functions $\Delta(T)$ and $F(\vartheta)$ depend on superfluidity type (e.g. Yakovlev et al. 1999). For cases A, B and C:

$F_{\mathrm{A}}(\vartheta)=1, \quad T_{\mathrm{cA}}=0.5669 \Delta(0) ;$
$F_{\mathrm{B}}(\vartheta)=1+3 \cos ^{2} \vartheta, T_{\mathrm{cB}}=0.8416 \Delta(0) ;$
$F_{\mathrm{C}}(\vartheta)=\sin ^{2} \vartheta, \quad T_{\mathrm{cC}}=0.4926 \Delta(0)$.

The gap amplitude $\Delta(T)$ is assumed to be governed by the standard equations of the BCS theory (e.g., Tamagaki 1970); $\Delta(0)$ is related to the critical temperature $T_{\mathrm{c}}$ as indicated above.

For further analysis it is convenient to introduce the dimensionless variables:

$$
\begin{aligned}
& z=\frac{\varepsilon-\mu}{T}=\operatorname{sign}(x) \sqrt{x^{2}+y^{2}}, \\
& x=\frac{\eta}{T}, \quad y=\frac{\delta}{T}, \quad v=\frac{\Delta(T)}{T} .
\end{aligned}
$$

While $T$ decreases from $T_{\mathrm{c}}$ to 0 , the parameter $v$ varies from 0 to $\infty$ as described, e.g., by Eq. (11) in Yakovlev et al. (1999).

We assume that the neutrino emissivity in superfluid matter can be calculated from Eqs. (10)-(12) by replacing $x_{j} \rightarrow z_{j}$ for all particle species which are in superfluid state. This assumption is widely used in the literature; its validity is discussed by Yakovlev et al. (2001). In this approximation, the neutrino emissivity of the modified Urca process can be written as

$Q^{\mathrm{n}}=Q_{0}^{\mathrm{n}} R^{\mathrm{n}}, \quad Q^{\mathrm{p}}=Q_{0}^{\mathrm{p}} R^{\mathrm{p}}$,

where $Q_{0}^{\mathrm{n}}$ and $Q_{0}^{\mathrm{p}}$ are the emissivities in a non-superfluid matter, while $R^{\mathrm{n}}$ and $R^{\mathrm{p}}$ are the factors which describe the reduction of the emissivity by nucleon superfluidity 
$\left(R^{\mathrm{N}}<1\right)$. Generally, these factors can be written as

$$
\begin{aligned}
R^{\mathrm{N}}= & \frac{J_{\mathrm{N}}}{I_{0}^{\mathrm{N}} A_{0}^{\mathrm{N}}} \\
J_{\mathrm{N}}= & 4 \pi \int \prod_{j=1}^{5} \mathrm{~d} \Omega_{j} \int_{0}^{\infty} \mathrm{d} x_{\nu} x_{\nu}^{3}\left[\prod_{j=1}^{5} \int_{-\infty}^{+\infty} \mathrm{d} x_{j} f\left(z_{j}\right)\right] \\
& \times \delta\left(x_{\nu}-\sum_{j=1}^{5} z_{j}\right) \delta\left(\sum_{j=1}^{5} \boldsymbol{p}_{j}\right)
\end{aligned}
$$

The notations are the same as in Eqs. (10)-(12).

We have composed a code which calculates the reduction factor (22) for proton superfluidity A and neutron superfluidity A, B, or C. The code has been tested by comparing with the analytical asymptotes at large $v_{1}$ and $v_{2}$ and with the results of Yakovlev \& Levenfish (1995) who considered superfluidity of either protons or neutrons. The results have also been compared with those calculated from Eq. (22) under simplified assumption $p_{\mathrm{Fp}}, p_{\mathrm{Fe}} \ll p_{\mathrm{Fn}}$ discussed below (see Eq. (41)).

Notice that the results of this section can also be used to describe modified Urca process with muons instead of electrons (see Yakovlev et al. 2001, for details).

\subsection{Reduction by superfluidity of neutrons and protons of type $\mathrm{A}$}

In this case Eq. (22) can be simplified. For pairing A, the dimensionless energy gap $y_{\mathrm{A}}$ is angle-independent. This allows one to decompose the integrals over the angles and over the dimensionless energies $x_{j}$. For the neutron branch of the modified Urca process we get

$$
\begin{aligned}
R_{\mathrm{AA}}^{\mathrm{n}}= & \frac{120960}{11513 \pi^{8}}\left[\prod_{j=1}^{4} \int_{-\infty}^{+\infty} \mathrm{d} x_{j} f\left(z_{j}\right)\right] \\
& \times G\left(z_{1}+z_{2}+z_{3}+z_{4}\right), \\
G(a) \equiv & \int_{-a}^{+\infty} \mathrm{d} x \frac{(x+a)^{3}}{1+e^{x}}
\end{aligned}
$$

where $j=1,2$, and 3 enumerates the reacting neutrons, while $j=4$ refers to a proton.

Let here and hereafter $v_{1} \equiv v_{\mathrm{n}}$ refer to neutrons, and $v_{2} \equiv v_{\mathrm{p}}$ refer to protons. The reduction of the proton branch is evidently given by

$R_{\mathrm{AA}}^{\mathrm{p}}\left(v_{1}, v_{2}\right)=R_{\mathrm{AA}}^{\mathrm{n}}\left(v_{2}, v_{1}\right)$.

It is useful to find the asymptotes of $R_{\mathrm{AA}}^{\mathrm{n}}$ from Eq. (23) for strong superfluidity, i.e., for large values of $v_{1}$ and $v_{2}$. In this case we can introduce three regions of the parameters (Fig. 1), where the asymptote of $R_{\mathrm{AA}}^{\mathrm{n}}$ has different forms. Region I corresponds to $v_{1}>v_{2}$; region II corresponds to $v_{2} \geq v_{1} \geq v_{2} / 3$; and region III corresponds to $v_{1}<$ $v_{2} / 3$. The asymptotes presented below are valid at $\left(v_{1}-\right.$ $\left.v_{2}\right)^{2} \gg v_{1}$ and $\left(v_{2}-3 v_{1}\right)^{2} \gg v_{2}$, i.e., not too close to the boundaries between regions I and II and between regions II and III.

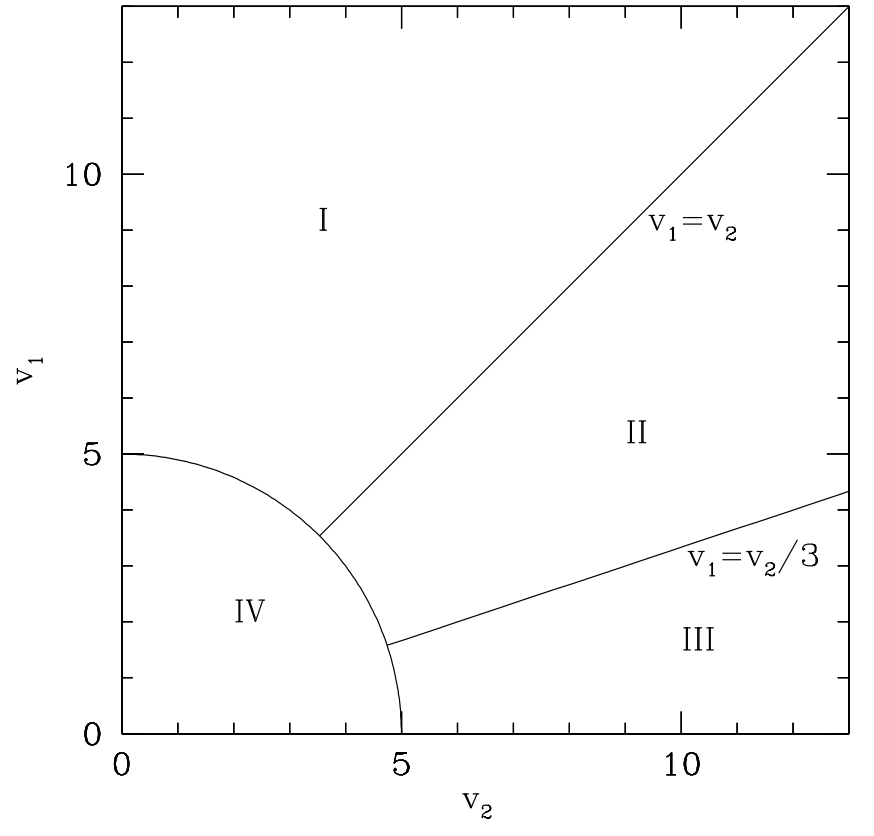

Fig. 1. Four regions of $v_{1}=v_{\mathrm{n}}$ and $v_{2}=v_{\mathrm{p}}$ where the reduction factors $R^{\mathrm{N}}$ of the neutron and proton branches of modified Urca process can be fitted by different expressions. In regions I, II, and III at $v_{1} \gg 1$ and $v_{2} \gg 1$ the factors $R^{\mathrm{n}}$ of neutron branch have different asymptotes.

For example, we outline the derivation of the asymptote of $R_{\mathrm{AA}}^{\mathrm{n}}$ from Eq. (23) in region I; the derivation in other regions is similar. Clearly, the integral (23) can be subdivided into several parts in such a way that any single part contains integrations from $-\infty$ to 0 and/or from 0 to $+\infty$. Now let us introduce the convenient notations for these parts. Let $R(2,-1)$ mean a five-dimensional integral containing the integration from 0 to $+\infty$ over two neutron variables, and over $-\infty$ to 0 over a proton variable (in this case, the integration over the third neutron variable is assumed to extend from $-\infty$ to 0 ). Splitting the initial integral (23) into the elementary integrals, we see that the same integral $R(2,-1)$ enters the sum three times. Thus, it is sufficient to calculate the integral once and multiply by 3 .

In this way we obtain eight integrals of different types: $R(3,+1), \quad R(3,-1), \quad R(2,+1), \quad R(2,-1)$, $R(1,+1), \quad R(1,-1), \quad R(0,+1)$, and $R(0,-1)$. In the limit of strong superfluidity $\left(v_{1,2} \gg 1\right)$, each of them is exponentially small. The exponentials are:

$$
\begin{array}{ll}
R(3,+1) \propto \exp \left(-3 v_{1}-v_{2}\right), & R(1,+1) \propto \exp \left(-2 v_{1}\right) \\
R(3,-1) \propto \exp \left(-3 v_{1}\right), & R(1,-1) \propto \exp \left(-2 v_{1}-v_{2}\right) \\
R(2,+1) \propto \exp \left(-2 v_{1}-v_{2}\right), & R(0,+1) \propto \exp \left(-3 v_{1}\right) \\
R(2,-1) \propto \exp \left(-2 v_{1}\right), & R(0,-1) \propto \exp \left(-3 v_{1}-v_{2}\right) .
\end{array}
$$

It is seen that the main contribution into the asymptote comes from $R(2,-1)$ and $R(1,+1)$. These terms have the same exponential but $R(2,-1)$ has a larger pre-exponent. Therefore, it is $R(2,-1)$ which gives the main contribution 
in region $\mathrm{I}$ :

$$
\begin{aligned}
R(2,-1)= & \frac{120960}{11513 \pi^{8}} \iint_{0}^{+\infty} \mathrm{d} x_{1} f\left(z_{1}\right) \mathrm{d} x_{2} f\left(z_{2}\right) \\
& \times \iint_{-\infty}^{0} \mathrm{~d} x_{3} f\left(z_{3}\right) \mathrm{d} x_{4} f\left(z_{4}\right) G(a),
\end{aligned}
$$

where $a=z_{1}+z_{2}+z_{3}+z_{4}$. One has $G(a) \rightarrow a^{4} / 4$ as $a \rightarrow \infty$ and $G(a) \rightarrow 6 \exp (a)$ as $a \rightarrow-\infty$. We are especially interested in large and positive $a$ for which $G(a) \approx a^{4} \theta(a) / 4$, where $\theta(x)$ is the step function. In this approximation, Eq. (26) can be rewritten as:

$$
\begin{aligned}
R(2,-1)= & \frac{120960}{11513 \pi^{8}} \iint_{0}^{+\infty} \mathrm{d} x_{1} f\left(z_{1}\right) \mathrm{d} x_{2} f\left(z_{2}\right) \\
& \times \iint_{-\infty}^{0} \mathrm{~d} x_{3} f\left(z_{3}\right) \mathrm{d} x_{4} f\left(z_{4}\right) \frac{a^{4}}{4} \theta(a) .
\end{aligned}
$$

Two integrals in Eq. (27) over the neutron variables $x_{1}$ and $x_{2}$ are rapidly converging because of the presence of exponentially decreasing functions. Accordingly, the region of space, where these two variables produce the main contribution, is rather small. Thus, it is sufficient to set $z_{1}=v_{1}$ and $z_{2}=v_{1}$ in the $\theta$ function. Then the integrations over $x_{1}$ and $x_{2}$ are separated and done analytically. Neglecting exponentially small terms while integrating over $x_{3}$ and $x_{4}$, we obtain the asymptote of $R_{\mathrm{AA}}^{\mathrm{n}}$ in region I:

$$
\begin{aligned}
R_{\mathrm{AA}}^{\mathrm{n}}= & 3 \frac{120960}{11513 \pi^{8}} \frac{\pi}{2} v_{1}^{6} v_{2} \mathrm{e}^{-2 v_{1}} I_{1}\left(\frac{v_{2}}{v_{1}}\right), \\
I_{1}(\alpha)= & \frac{1}{4} \iint_{0}^{+\infty} \mathrm{d} x_{3} \mathrm{~d} x_{4}\left(2-r_{3}-\alpha r_{4}\right)^{4} \\
& \times \theta\left(2-r_{3}-\alpha r_{4}\right), \quad 0<\alpha \leq 1,
\end{aligned}
$$

where $r_{j}=\sqrt{x_{j}^{2}+1}$.

In the same manner in region II we obtain:

$$
\begin{aligned}
R_{\mathrm{AA}}^{\mathrm{n}}= & 3 \frac{120960}{11513 \pi^{8}} \frac{\pi}{2} v_{1}^{13 / 2} v_{2}^{1 / 2} \mathrm{e}^{-v_{1}-v_{2}} I_{2}\left(\frac{v_{2}}{v_{1}}\right) \\
& +\frac{120960}{11513 \pi^{8}}\left(\frac{\pi}{2}\right)^{3 / 2} v_{1}^{3 / 2} \mathrm{e}^{-3 v_{1}} K \\
I_{2}(\alpha)= & \frac{1}{4} \iint_{0}^{+\infty} \mathrm{d} x_{1} \mathrm{~d} x_{2}\left(1+\alpha-r_{1}-r_{2}\right)^{4} \\
& \times \theta\left(1+\alpha-r_{1}-r_{2}\right), \quad 1 \leq \alpha \leq 3 \\
K= & \frac{\sqrt{9 v_{1}^{2}-v_{2}^{2}}}{120}\left(486 v_{1}^{4}+747 v_{1}^{2} v_{2}^{2}+16 v_{2}^{4}\right) \\
& -\frac{3}{8} v_{1} v_{2}^{2}\left(3 v_{2}^{2}+36 v_{1}^{2}\right) \ln \frac{3 v_{1}+\sqrt{9 v_{1}^{2}-v_{2}^{2}}}{v_{2}} .
\end{aligned}
$$

In region III:

$$
\begin{aligned}
R_{\mathrm{AA}}^{\mathrm{n}}= & \frac{120960}{11513 \pi^{8}}\left(\frac{\pi}{2}\right)^{1 / 2} v_{1}^{3} v_{2}^{9 / 2} \mathrm{e}^{-v_{2}} I_{3}\left(\frac{v_{2}}{v_{1}}\right), \\
I_{3}(\alpha)= & \frac{1}{4} \iiint_{0}^{+\infty} \mathrm{d} x_{1} \mathrm{~d} x_{2} \mathrm{~d} x_{3}\left[1-\frac{1}{\alpha}\left(r_{1}+r_{2}+r_{3}\right)\right]^{4} \\
& \times \theta\left(\alpha-r_{1}-r_{2}-r_{3}\right),
\end{aligned}
$$

where $3 \leq \alpha<\infty$.
The asymptotes themselves contain complicated integrals. Thus we have calculated the asymptotes of the integrals (29) and (34). In region I we have:

$\begin{array}{ll}I_{1}=\frac{0.027570965}{\alpha} & \alpha \rightarrow 0, \\ I_{1}=0.0785398(1-\alpha)^{5} & \alpha \rightarrow 1 .\end{array}$

The asymptote of $I_{2}(\alpha)$ at $\alpha \rightarrow 1$ can be determined by matching the reduction factors at the boundary of regions I and II.

In region III we have

$I_{3}=0.214307\left(1-\frac{3}{\alpha}\right)^{11 / 2} \quad \alpha \rightarrow 3$,

$I_{3}=\frac{1}{840} \alpha^{3} \quad \alpha \rightarrow \infty$.

We have numerically calculated the functions $I_{1}(\alpha), I_{2}(\alpha)$, and $I_{3}(\alpha)$ and proposed analytic fits which reproduce the results and the asymptotes (29), (31), and (34) with the maximum error less than $1 \%$. For region I, the fit is:

$I_{1} \approx \frac{0.02757096\left(1-\alpha^{p_{1}}\right)^{5}}{\alpha\left(1+30 \alpha^{2}+60 \alpha^{4}+107.186 \alpha^{6}\right)^{p_{2}}}$,

where $p_{1}=1.473$ and $p_{2}=0.1684$.

For region II:

$I_{2} \approx 0.05\left(\alpha^{p_{1}}-1\right)^{5}\left(\alpha^{p_{2}}-p_{3}\right)^{2}$

with $p_{1}=1.210, p_{2}=0.222$, and $p_{3}=0.215$.

For region III:

$I_{3} \approx \frac{\alpha^{3}}{840}\left(1-\frac{9}{\alpha^{2}}\right)^{5.5}\left(1+\frac{p_{1}}{\alpha^{p_{2}}}\right)^{-p_{3}}$,

where $p_{1}=8.363, p_{2}=1.427$, and $p_{3}=1.8978$.

The fits of $R_{\mathrm{AA}}^{\mathrm{n}}$ are given in Appendix.

\subsection{The neutron branch reduced by proton superfluidity $\mathrm{A}$ and neutron superfluidity $\mathrm{B}$}

Now consider neutron superfluidity of type B. According to Eqs. (20) and (18), the dimensionless energy gap of the neutrons, $y_{\mathrm{B}}$, is angle-dependent. The asymptotes of $R_{\mathrm{AB}}^{\mathrm{n}}$ in this case can be obtained from Eq. (22). As before, $j=1,2$, and 3 enumerates neutrons while $j=4$ refers to a proton. Equation (22) can be written as

$$
\begin{aligned}
R_{\mathrm{AB}}^{\mathrm{n}}= & \frac{120960}{11513 \pi^{8}} \frac{1}{(4 \pi)^{3}} \frac{p_{\mathrm{Fn}}^{3}}{p_{\mathrm{Fe}} p_{\mathrm{Fp}}} \int \frac{\mathrm{d} \Omega_{1} \mathrm{~d} \Omega_{2} \mathrm{~d} \Omega_{3}}{\left|\boldsymbol{p}_{\mathrm{Fn} 1}+\boldsymbol{p}_{\mathrm{Fn} 2}+\boldsymbol{p}_{\mathrm{Fn} 3}\right|} \\
& \times I \theta\left(\boldsymbol{p}_{\mathrm{Fn} 1}+\boldsymbol{p}_{\mathrm{Fn} 2}+\boldsymbol{p}_{\mathrm{Fn} 3}\right), \\
I= & {\left[\prod_{j=1}^{4} \int_{-\infty}^{+\infty} \mathrm{d} x_{j} f\left(z_{j}\right)\right] G\left(z_{1}+z_{2}+z_{3}+z_{4}\right), \quad(40) }
\end{aligned}
$$

where $G(a)$ is given by Eq. (24); $\theta$ is the step function: $\theta=1$ if $\left|p_{\mathrm{Fe}}-p_{\mathrm{Fp}}\right| \leq\left|\boldsymbol{p}_{\mathrm{Fn} 1}+\boldsymbol{p}_{\mathrm{Fn} 2}+\boldsymbol{p}_{\mathrm{Fn} 3}\right| \leq p_{\mathrm{Fe}}+p_{\mathrm{Fp}}$, and $\theta=0$ otherwise. 
Calculations show that the reduction factor is almost insensitive to variations of particle Fermi-momenta. Let us obtain a simplified expression for $R_{\mathrm{AB}}^{\mathrm{n}}$ by setting $p_{\mathrm{Fp}}=$ $p_{\mathrm{Fe}}=0$ in Eq. (22) and integrating over $\mathrm{d} \Omega_{\mathrm{p}}$ and $\mathrm{d} \Omega_{\mathrm{e}}$. The result is

$$
\begin{aligned}
R_{\mathrm{AB}}^{\mathrm{n}}= & \frac{120960}{11513 \pi^{8}} \frac{p_{\mathrm{Fn}}^{3}}{2(2 \pi)^{2}} \int \prod_{j=1}^{3} \mathrm{~d} \Omega_{j} \delta\left(\sum_{j=1}^{3} \boldsymbol{p}_{j}\right) \\
& \times\left[\prod_{j=1}^{5} \int_{-\infty}^{+\infty} \mathrm{d} x_{j} f\left(z_{j}\right)\right] G\left(z_{1}+z_{2}+z_{3}+z_{4}\right) .
\end{aligned}
$$

As for neutron superfluidity A, we have different asymptotes of $R_{\mathrm{AB}}^{\mathrm{n}}$ in regions I, II, and III. For example, consider the asymptote at large $v_{1}$ and $v_{2}$ in region I. It is easy to see that the main contribution comes from $R(2,-1)$ (see Eq. (27)). In Eq. (27) it is sufficient to set $z_{j}=\operatorname{sign}\left(x_{j}\right) \sqrt{x_{j}^{2}+v_{1}^{2}\left(1+3 c_{j}^{2}\right)}$ (for $j=1,2,3$ ) and $z_{4}=\operatorname{sign}\left(x_{4}\right) \sqrt{x_{4}^{2}+v_{2}^{2}}$. Here, $c_{j} \equiv \cos \vartheta_{j}$. The simplified reduction factor will then be rewritten as:

$$
\begin{aligned}
& R_{\mathrm{AB}}^{\mathrm{n}}=\frac{3}{2(2 \pi)^{2}} \int_{-1}^{1} \mathrm{~d} c_{1} \mathrm{~d} c_{2} \mathrm{~d} c_{3} D\left(c_{1}, c_{2}, c_{3}\right) R(2,-1), \\
& D\left(c_{1}, c_{2}, c_{3}\right) \equiv \int_{0}^{2 \pi} \mathrm{d} \varphi_{1} \mathrm{~d} \varphi_{2} \mathrm{~d} \varphi_{3} \delta\left(\sum_{j=1}^{3} \frac{\boldsymbol{p}_{j}}{p_{\mathrm{Fn}}}\right) \\
& =\frac{4 \pi \theta\left(0.75-c_{1} c_{2}-c_{1}^{2}-c_{2}^{2}\right)}{\sqrt{0.75-c_{1} c_{2}-c_{1}^{2}-c_{2}^{2}}} \delta\left(c_{1}+c_{2}+c_{3}\right) .
\end{aligned}
$$

Notice that the main contribution into (42) comes from the range of angles $c_{1} \approx c_{2} \approx 0$. Then it is sufficient to set $z_{i} \approx v_{1}+0.5 x_{i}^{2} v_{1}^{-1}+1.5 c_{i}^{2} v_{1}(i=1,2)$ in the exponentials in $R(2,-1)$, and $z_{1}=z_{2}=v_{1}$ in all other functions. This leads to the following asymptote in region I:

$R_{\mathrm{AB}}^{\mathrm{n}}=3 \frac{120960}{11513 \pi^{8}} \frac{\pi}{3 \sqrt{3}} v_{1}^{5} v_{2} \mathrm{e}^{-2 v_{1}} I_{1}\left(\frac{v_{2}}{v_{1}}\right)$,

where $I_{1}(\alpha)$ is defined by Eq. (29). Now let us introduce three functions $I_{i}\left(c_{1}, c_{2}, c_{3}, \alpha\right)(i=1,2$, and 3$)$, which formally coincide with those given by Eqs. (29), (31), and (34) with the only difference that now $r_{j}=$ $\sqrt{x_{j}^{2}+1+3 c_{j}^{2}}, j=1,2$, and 3 .

Then, the asymptote in region II will be written as

$$
\begin{aligned}
R_{\mathrm{AB}}^{\mathrm{n}}= & 3 \frac{120960}{11513 \pi^{8}} \frac{\pi^{-1 / 2}}{8 \sqrt{6}} v_{1}^{6} v_{2}^{1 / 2} \mathrm{e}^{-v_{1}-v_{2}} \\
& \times \int_{-1}^{1} \mathrm{~d} c_{1} \mathrm{~d} c_{2} D\left(c_{1}, c_{2}, 0\right) I_{2}\left(c_{1}, c_{2}, 0, \frac{v_{2}}{v_{1}}\right) \\
& +\frac{120960}{11513 \pi^{8}} \frac{\pi^{3 / 2}}{3 \sqrt{6}} v_{1}^{1 / 2} \mathrm{e}^{-3 v_{1}} K,
\end{aligned}
$$

where $K$ is given by Eq. (32). In region III we obtain:

$$
\begin{aligned}
R_{\mathrm{AB}}^{\mathrm{n}}= & \frac{120960}{11513 \pi^{8}} \frac{\pi^{-3 / 2}}{8 \sqrt{2}} v_{1}^{3} v_{2}^{9 / 2} \mathrm{e}^{-v_{2}} \\
& \times \int_{-1}^{1} \mathrm{~d} c_{1} \mathrm{~d} c_{2} \mathrm{~d} c_{3} D\left(c_{1}, c_{2}, c_{3}\right) I_{3}\left(c_{1}, c_{2}, c_{3}, \frac{v_{2}}{v_{1}}\right) .
\end{aligned}
$$

We have numerically integrated $R_{\mathrm{AB}}^{\mathrm{n}}$ from Eq. (40) for a dense grid of $v_{1}$ and $v_{2}$. The calculations have been conducted at $p_{\mathrm{Fp}}=0.11 p_{\mathrm{Fn}}$ and $p_{\mathrm{Fe}}=0.1 p_{\mathrm{Fn}}$. As mentioned above, the reduction factor is rather insensitive to variations of these parameters. The variations of $R_{\mathrm{AB}}^{\mathrm{n}}$ to the changes of the particle Fermi momenta within reasonable limits $\left(p_{\mathrm{Fe}, \mathrm{p}} \leq(0.3-0.4) p_{\mathrm{Fn}}\right)$ obtained in some test runs are of the order of estimated error of numerical integration. The fits of $R_{\mathrm{AB}}^{\mathrm{n}}$ are given in Appendix.

\subsection{The proton branch reduced by proton superfluidity A and neutron superfluidity B}

In this case Eq. (22) can be simplified as

$R_{\mathrm{AB}}^{\mathrm{p}}=\frac{120960}{11513 \pi^{8}} \frac{1}{2} \int_{-1}^{1} \mathrm{~d} c_{4} I\left(z_{1}, z_{2}, z_{3}, z_{4}\right)$.

Here, $c_{4} \equiv \cos \vartheta_{4}$. The function $I\left(z_{1}, z_{2}, z_{3}, z_{4}\right)$ is defined by Eq. (40) with the only difference that now $j=1,2$, and 3 refer to protons, while $j=4$ refers to a neutron.

One can easily obtain the asymptotes of the reduction factor at large values of $v_{1}$ and $v_{2}$. For the proton branch of modified Urca process, the regions where the asymptotes are different can be found from neutron-branch regions by replacing $v_{1} \rightleftharpoons v_{2}$. For instance, at $v_{2}>v_{1}$ :

$R_{\mathrm{AB}}^{\mathrm{p}}=3 \frac{120960}{11513 \pi^{8}} \frac{\pi}{4} v_{1} v_{2}^{6} \mathrm{e}^{-2 v_{2}} \int_{-1}^{1} \mathrm{~d} c_{4} I_{1}\left(c_{4}, \frac{v_{1}}{v_{2}}\right)$,

where $I_{1}\left(c_{4}, \alpha\right)$ is defined by Eq. (29) with $r_{4}=$ $\sqrt{x_{4}^{2}+1+3 c_{4}^{2}}$. At $v_{1} \geq v_{2} \geq v_{1} / 3$ :

$$
\begin{aligned}
R_{\mathrm{AB}}^{\mathrm{p}}= & 3 \frac{120960}{11513 \pi^{8}}\left(\frac{\pi}{2}\right)^{3 / 2} v_{2}^{13 / 2} \mathrm{e}^{-v_{1}-v_{2}} I_{2}\left(\frac{v_{1}}{v_{2}}\right) \\
& +\frac{120960}{11513 \pi^{8}} \frac{\pi^{3 / 2}}{2^{9 / 2}} v_{1} v_{2}^{11 / 2} \mathrm{e}^{-3 v_{2}} \int_{-1}^{1} \mathrm{~d} c_{4} \\
& \times \int_{0}^{\infty} \mathrm{d} x_{4}\left(3-\frac{v_{1}}{v_{2}} r_{4}\right)^{4} \theta\left(3-\frac{v_{1}}{v_{2}} r_{4}\right) .
\end{aligned}
$$

At $v_{2}<v_{1} / 3$ :

$R_{\mathrm{AB}}^{\mathrm{p}}=\frac{120960}{11513 \pi^{8}} \frac{\pi}{2 \sqrt{3}} v_{1}^{4} v_{2}^{3} \mathrm{e}^{-v_{1}} I_{3}\left(\frac{v_{1}}{v_{2}}\right)$.

The fits of $R_{\mathrm{AB}}^{\mathrm{p}}$ are given in Appendix.

\subsection{The neutron branch reduced by proton superfluidity $\mathrm{A}$ and neutron superfluidity $\mathrm{C}$}

The most important feature of this case is that the energy gap vanishes at the poles of the Fermi sphere (see Eqs. (19) and (20)). Equation (40) remains valid in this case. The calculations of $R_{\mathrm{AC}}^{\mathrm{n}}$ have been done at $p_{\mathrm{Fp}}=0.11 p_{\mathrm{Fn}}$ and $p_{\mathrm{Fe}}=0.1 p_{\mathrm{Fn}}$. As in the previous cases, the reduction factor is rather insensitive to variations of these parameters. The results are approximated by the expressions given in Appendix. 


\subsection{The proton branch reduced by proton superfluidity A and neutron superfluidity $\mathrm{C}$}

Equation (46) remains valid in this case. Since $y_{4}=y_{\mathrm{C}}=$ $v_{1} \sin \vartheta_{4}$ vanishes at the poles of the Fermi sphere, the reduction factor $R_{\mathrm{AC}}^{\mathrm{p}}$ varies with $v_{1}$ as a power-law (rather than exponentially). It is easy to determine its behavior at large $v_{1}$. One can see that in this case the main contribution into integral (46) comes from the region where $\sin \vartheta_{4} \ll 1$. Thus, we have

$R_{\mathrm{AC}}^{\mathrm{p}}=\frac{120960}{11513 \pi^{8}} v_{1}^{-2} \int_{0}^{+\infty} \mathrm{d} t t I\left(z_{1}, z_{2}, z_{3}, z_{4}\right)$.

Here, $I$ is given by Eq. (40), in which $z_{4}=$ $\operatorname{sign}\left(x_{4}\right) \sqrt{x_{4}^{2}+t^{2}}$. The difference of exact Eq. (46) from Eq. (50) is less than $2 \%$ at $v_{1} \gtrsim 25$. As in the previous cases, $R_{\mathrm{AC}}^{\mathrm{p}}$ has been calculated numerically. For $v_{1} \geq 25$, we have used Eq. (50) and fitted our results by a simple equation

$$
\begin{aligned}
R_{\mathrm{AC}}^{\mathrm{p}} & =v_{1}^{-2} \exp \left(p_{4}-\frac{p_{1} v_{2}^{2}}{\left(1+p_{2} v_{2}^{2}+p_{5} v_{2}^{4}\right)^{p_{3}}}\right), \\
p_{1} & =0.289297, \quad p_{2}=0.152895, \quad p_{3}=0.228536, \\
p_{4} & =2.250496, \quad p_{5}=3.885153 \times 10^{-3} .
\end{aligned}
$$

The fits of $R_{\mathrm{AC}}^{\mathrm{p}}$ for $v_{1} \leq 25$ are given in Appendix.

\section{Approximate reduction factors of the NN-bremsstrahlung in superfluid matter}

Now consider the superfluid suppression of the neutrinopair emission in the nucleon-nucleon bremsstrahlung processes (4)-(6). In the absence of superfluidity the emissivities of NN-bremsstrahlung processes in the onepion-exchange approximation are given, for instance, by Yakovlev et al. (1999).

In analogy to Eq. (21), one can introduce the superfluid reduction factors $R^{\mathrm{NN}}$ :

$Q_{\mathrm{NN}}=Q_{\mathrm{NN} 0} R^{\mathrm{NN}}$,

where

$$
\begin{aligned}
R^{\mathrm{NN}}= & \frac{945}{164 \pi^{8}} \frac{p_{\mathrm{Fn}}^{3}}{32 \pi^{3}} \int \prod_{j=1}^{4} \mathrm{~d} \Omega_{j} \delta\left(\sum_{j=1}^{4} \boldsymbol{p}_{j}\right) I^{\mathrm{NN}}, \\
I^{\mathrm{NN}}= & \int_{0}^{\infty} \mathrm{d} x_{\nu} x_{\nu}^{4}\left[\prod_{j=1}^{4} \int_{-\infty}^{+\infty} \mathrm{d} x_{j} f\left(z_{j}\right)\right] \\
& \times \delta\left(z_{1}+z_{2}+z_{3}+z_{4}-x_{\nu}\right) .
\end{aligned}
$$

Here, $z_{j}$ is given by Eq. (20), and $j$ enumerates initialstate and final-state particles participating in the reactions. Accordingly, if we consider neutron superfluidity $\mathrm{C}$, and proton superfluidity $\mathrm{A}$, then we have $z_{j}=\operatorname{sign}\left(x_{j}\right) \sqrt{x_{j}^{2}+v_{1}^{2} \sin ^{2} \vartheta_{j}}$ for neutrons, and $z_{j}=$ $\operatorname{sign}\left(x_{j}\right) \sqrt{x_{j}^{2}+v_{2}^{2}}$ for protons.

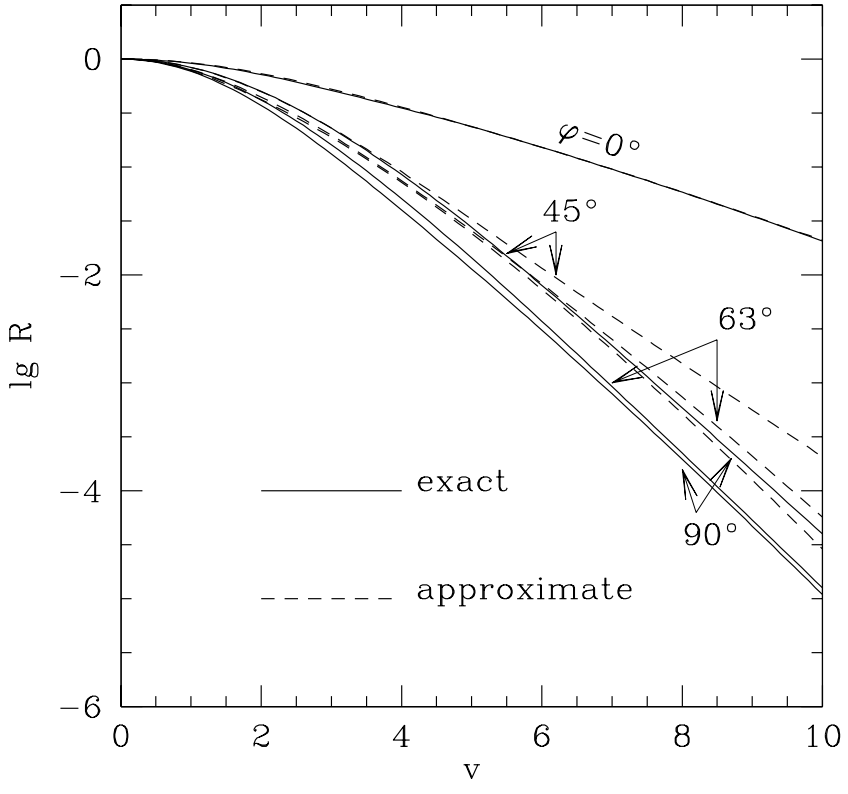

Fig. 2. Reduction of the neutrino emissivity by neutron and proton superfluidities of type A in the neutron branch of the modified Urca process versus $v\left(v_{1}=v \sin \varphi, v_{2}=v \cos \varphi\right)$ at $\varphi=0,45,63$, and $90^{\circ}$. Solid lines show our results, and dashed lines are obtained from similarity criteria, e.g., Yakovlev et al. (1999).

The factor $R_{\mathrm{AA}}^{\mathrm{pp}}$ was accurately calculated by Yakovlev \& Levenfish (1995). For the neutron-proton process we suggest the similarity relation of the form

$R_{\mathrm{AC}}^{\mathrm{np}} \approx \frac{R_{\mathrm{AC}}^{\mathrm{D}}\left(v_{1}, v_{2}\right)}{R_{\mathrm{A}}^{\mathrm{D}}\left(v_{2}\right)} R_{\mathrm{pA}}^{\mathrm{np}}\left(v_{2}\right)$.

Here, $R_{\mathrm{AC}}^{\mathrm{D}}\left(v_{1}, v_{2}\right)$ is the reduction factor of the direct Urca process determined by Levenfish \& Yakovlev (1994). The subscript pA means that superfluidity of protons is of type A (and neutrons are non-superfluid). The factor $R_{\mathrm{pA}}^{\mathrm{np}}$ was calculated by Yakovlev \& Levenfish (1995).

An analysis of $R_{\mathrm{nC}}^{\mathrm{nn}}$ for neutron-neutron bremsstrahlung, Eq. (4), is more sophisticated (since no similarity criterion can be formulated). Let us study the reduction factor $R_{\mathrm{nC}}^{\mathrm{nn}}$ at large $v_{1}$ from Eq. (53). Now $j=1-4$ refer to neutrons. One can see that the main contribution to $R_{\mathrm{nC}}^{\mathrm{nn}}$ comes from the range of angles $\sin \vartheta_{j} \approx 0$. Since the sum of the Fermi momenta of reacting neutrons must be equal to zero, the Fermi momenta should concentrate to the poles of the Fermi sphere: two momenta to one pole and other two momenta to the other pole. Now we expand all functions in series over $\vartheta_{j}$ and integrate over $\varphi_{4}, \varphi_{3}, \varphi_{1}$, and $\vartheta_{4}$. In this way we obtain

$$
\begin{aligned}
R_{\mathrm{nC}}^{\mathrm{nn}}= & \frac{945}{164 \pi^{8}} \frac{96 \pi}{32 \pi^{3}} \int_{0}^{1} \mathrm{~d} \vartheta_{1} \mathrm{~d} \vartheta_{2} \mathrm{~d} \vartheta_{3} \vartheta_{1} I^{\mathrm{nn}} \\
& \times \theta\left(\vartheta_{2}^{2}+\vartheta_{3}^{2}-\vartheta_{1}^{2} \geq 0\right) \\
& \times \int_{0}^{1} \frac{\mathrm{d} t}{\sqrt{\left(1-t^{2}\right)\left(m^{2}-t^{2}\right)}} \theta(t \leq m), \\
m= & \frac{\vartheta_{1} \sqrt{\vartheta_{2}^{2}+\vartheta_{3}^{2}-\vartheta_{1}^{2}}}{\vartheta_{2} \vartheta_{3}}
\end{aligned}
$$




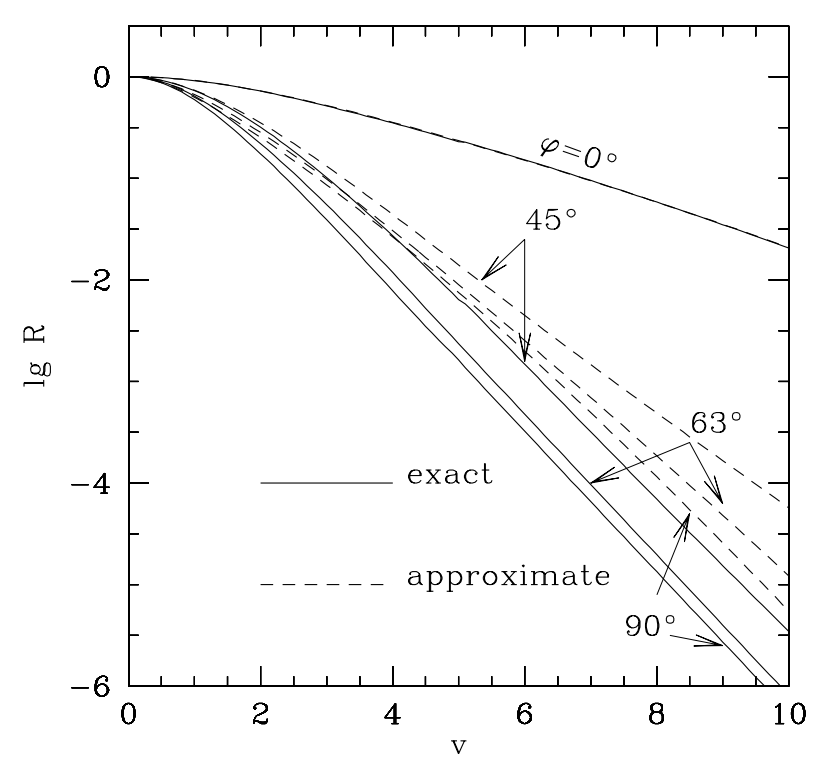

Fig. 3. Same as in Fig. 2 but for $R_{\mathrm{AB}}^{\mathrm{n}}$.

In Eq. (55) we introduce $t=\cos \varphi_{2} ; I^{\mathrm{nn}}$ is the same as in Eq. (53) with the only difference that now $\vartheta_{4}=$ $\sqrt{\vartheta_{2}^{2}+\vartheta_{3}^{2}-\vartheta_{1}^{2}}$. Introducing $y_{j}=v_{1} \vartheta_{j}, j=1,2,3$, and taking into account that $v_{1}$ is large, we can extend the upper limit of integration over $y_{j}$ to infinity. As a result, the asymptote of $R_{\mathrm{nC}}^{\mathrm{nn}}$ becomes

$$
\begin{aligned}
R_{\mathrm{nC}}^{\mathrm{nn}}= & \frac{945}{164 \pi^{8}} \frac{96 \pi}{32 \pi^{3}} v_{1}^{-4} \int_{0}^{\infty} \mathrm{d} y_{1} \mathrm{~d} y_{2} \mathrm{~d} y_{3} y_{1} I^{\mathrm{nn}} \\
& \times \theta\left(y_{2}^{2}+y_{3}^{2}-y_{1}^{2} \geq 0\right) \\
& \times \int_{0}^{1} \frac{\mathrm{d} t}{\sqrt{\left(1-t^{2}\right)\left(m^{2}-t^{2}\right)}} \theta(t \leq m) .
\end{aligned}
$$

Evaluating this integral, we obtain:

$R_{\mathrm{nC}}^{\mathrm{nn}}=\frac{11.533}{v_{1}^{4}}$

Let us derive an approximate formula for $R_{\mathrm{nC}}^{\mathrm{nn}}$ at intermediate values of $v_{1}$. For this purpose we substitute $\frac{f}{4}\left(y_{1}+y_{2}+y_{3}+y_{4}\right)$, where $y_{j}=v_{1} \sin \vartheta_{j}$, in the argument of the function $R_{\mathrm{nA}}^{\mathrm{nn}}\left(v_{1}\right)$ (as described by Yakovlev et al. 1999) and integrate this function over $\vartheta_{j}$ :

$R_{\mathrm{nC}}^{\mathrm{nn}} \approx \prod_{j=1}^{4}\left[\int_{0}^{1} \mathrm{~d} \sin \vartheta_{j}\right] R_{\mathrm{nA}}^{\mathrm{nn}}\left(\frac{f}{4}\left(y_{1}+y_{2}+y_{3}+y_{4}\right)\right)$,

where $f=2.248$ is chosen to satisfy the asymptote (58). For $v_{1} \leq 25, R_{\mathrm{nC}}^{\mathrm{nn}}$ has been calculated numerically. At $v_{1} \gtrsim 25$ our numerical results agree with the asymptote (58) within $1 \%$. The numerical results for $v_{1} \leq 25$ can be fitted as

$R_{\mathrm{nC}}^{\mathrm{nn}} \approx \exp \left(-\frac{p_{1} v_{1}^{2}}{\left(1+p_{2} v_{1}^{2}+p_{4} v_{1}^{4}\right)^{p_{3}}}\right)$,

where $p_{1}=0.442995, p_{2}=0.250953, p_{3}=0.410517, p_{4}=$ $7.09171 \times 10^{-3}$. The calculation and fit error does not exceed $2 \%$.

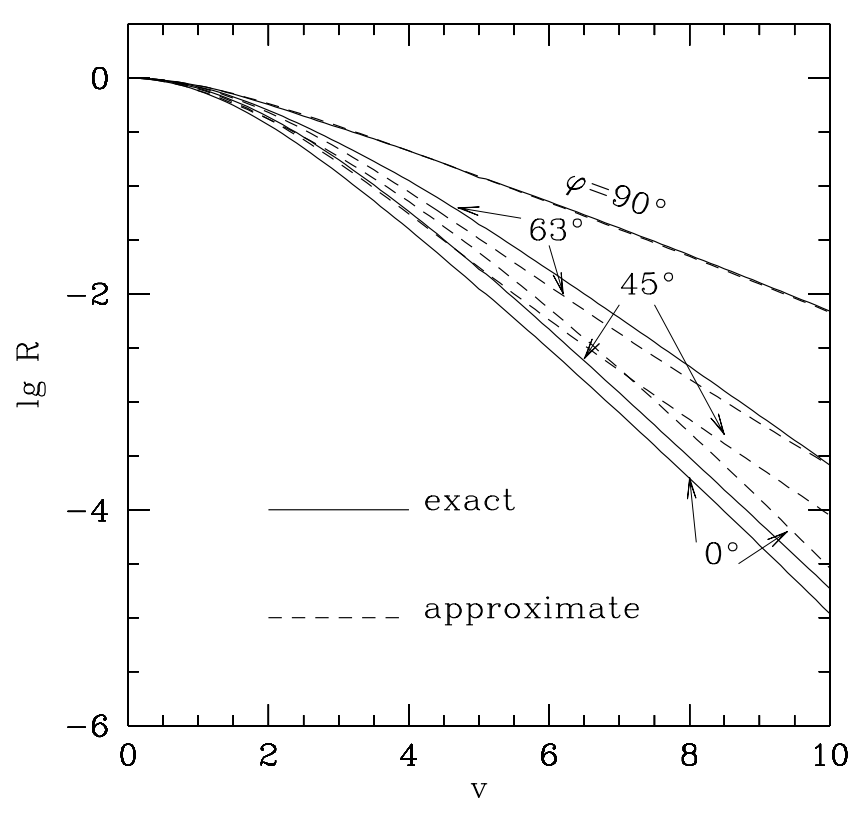

Fig. 4. Same as in Fig. 2 but for $R_{\mathrm{AB}}^{\mathrm{p}}$.

\section{Discussion}

The results of Sect. 2 allow us to compare the exact and approximate reduction factors of modified Urca process. The comparison is illustrated in Figs. 2-4. The figures show the dependence of the calculated reduction factors, $R_{\mathrm{AA}}^{\mathrm{n}}, R_{\mathrm{AB}}^{\mathrm{n}}$, and $R_{\mathrm{AB}}^{\mathrm{p}}$, on $v=\sqrt{v_{1}^{2}+v_{2}^{2}}$ at several values of $\varphi\left(\varphi\right.$ is the polar angle in the $v_{1}-v_{2}$ plane; $\left.\tan (\varphi)=\left(v_{1} / v_{2}\right)\right)$. Our results (solid lines) are compared with the approximate reduction factors (dashed lines) constructed (e.g., Yakovlev et al. 1999) using the criteria of similarity between the reduction factors for different neutrino reactions. The approximate factors have been used in a number of simulations of neutron star cooling. One can see that the difference of the approximate reduction factors from the exact ones increases with increasing $v$ (but for $\varphi=0^{\circ}$ in Figs. 2, 3 and $\varphi=90^{\circ}$ in Fig. 4).

Now let us answer the question which neutrino generation mechanism dominates in a superfluid neutron-star core. Taking into account the above results we can calculate the emissivities of all main neutrino processes (Sect. 1) for proton superfluidity A and any neutron superfluidity, A, B, or C. Figures 5 and 6 show which process dominates at different values of $T_{c n}$ and $T_{c \mathrm{p}}$. Figure 5 shows the effect of neutron superfluidity $B$, while Fig. 6 - the effect of neutron superfluidity C. Both figures are plotted using an equation of state suggested by Prakash et al. (1988) (their model I of symmetry energy with the compression modulus of saturated nuclear matter $K=240 \mathrm{MeV}$ ).

Three left panels of Fig. 5 illustrate standard neutrino emission at $\rho=2 \rho_{0}$ (direct Urca process forbidden) for three values of the internal stellar temperature, $T=10^{8}, 3 \times 10^{8}$, and $10^{9} \mathrm{~K}$, while three right panels correspond to neutrino emission enhanced by direct Urca process at $\rho=5 \rho_{0}$ for the same $T$. Figure 5 is almost the same as obtained earlier by Yakovlev et al. (1999) for another 

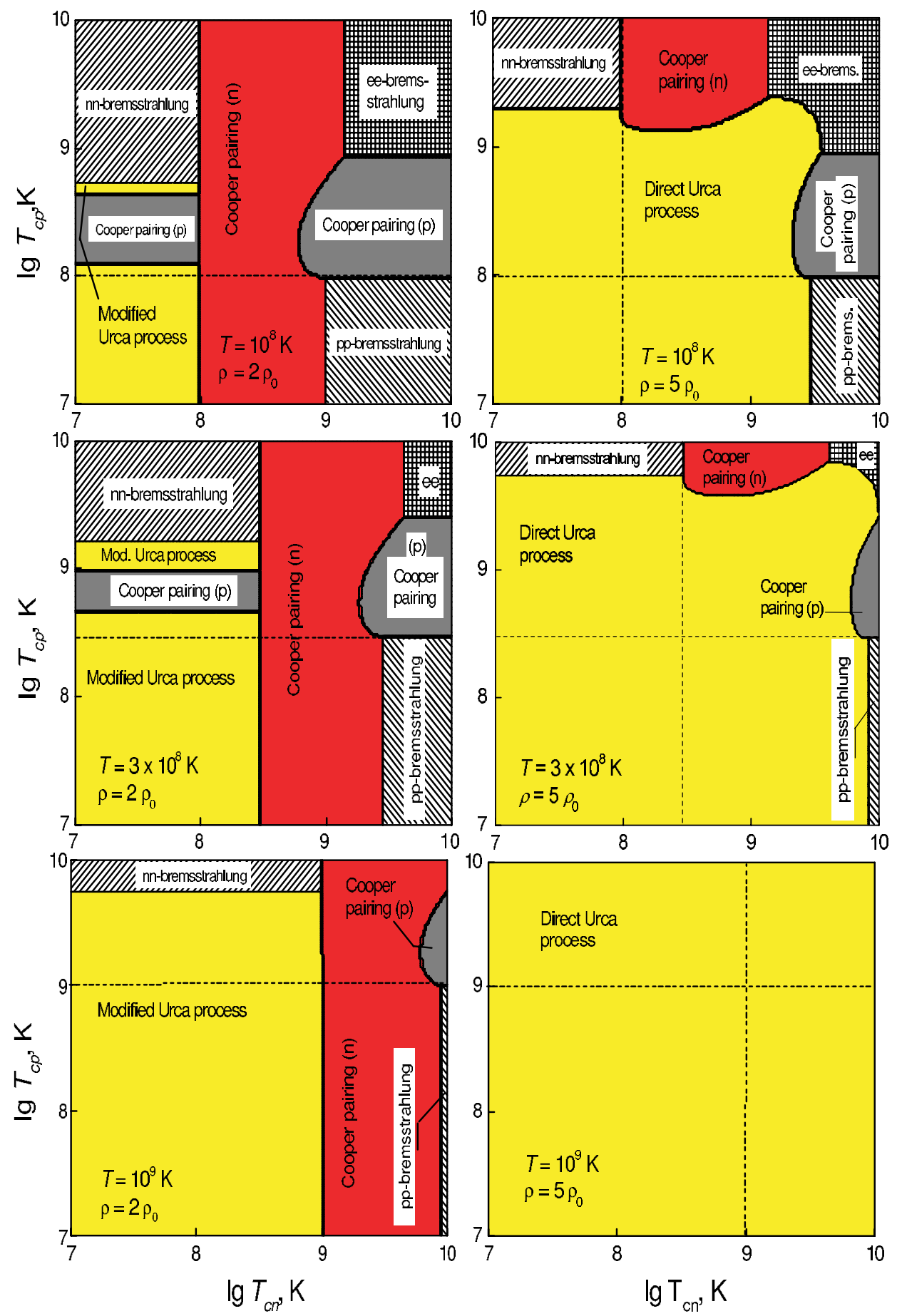

Fig. 5. Regions of $T_{\mathrm{cn}}$ (of type B) and $T_{\mathrm{cp}}$ (of type A) where the different neutrino reactions dominate.

equation of state using the approximate reduction factors of modified Urca process. The selected values of $T$ cover the temperature interval most important for the theory of neutron star cooling. The figures are almost independent of $\rho$ (and of equation of state) as long as $\rho$ does not cross the density threshold of opening direct Urca process. One can see that if the neutrons are superfluid alone and $T \ll T_{\mathrm{cn}}$, then the bremsstrahlung due to protonproton scattering becomes dominant. If protons are superfluid alone and $T \ll T_{\mathrm{cp}}$, then the main mechanism is neutrino emission in neutron-neutron bremsstrahlung. Neutrino emission due to Cooper pairing of neutrons always dominates at $T \sim 0.4 T_{\text {cn }}$ provided direct Urca process is forbidden. If the direct Urca process is allowed then the Cooper-pairing neutrino emission may dominate provided nucleons of one species are strongly superfluid while nucleons of the other species are moderately superfluid. Finally, in the presence of strong superfluidity of protons and neutrons, all the processes involving nucleons are so strongly reduced that the neutrino-pair emission in 

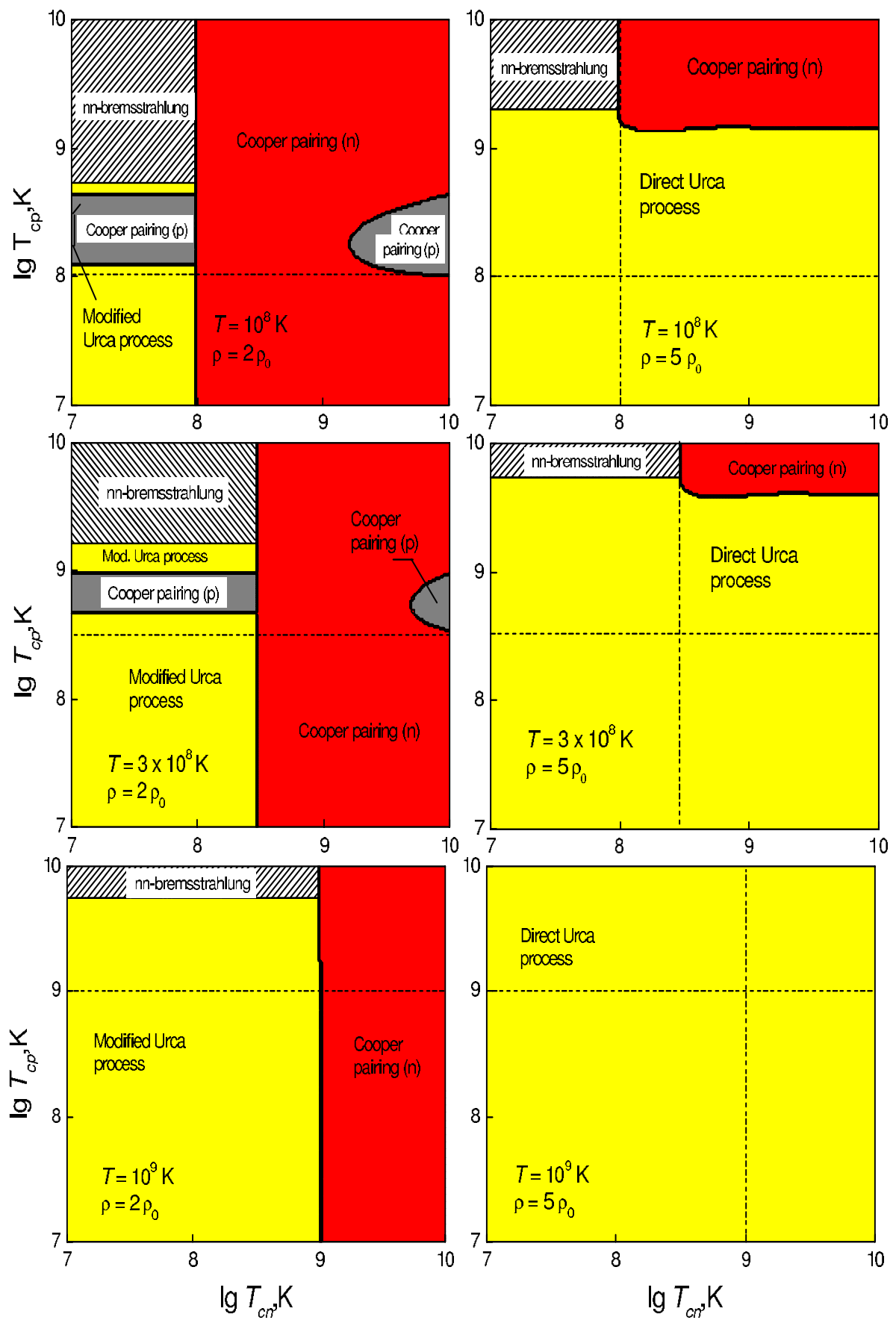

Fig. 6. Regions of $T_{\mathrm{cn}}$ (of type $\mathrm{C}$ ) and $T_{\mathrm{cp}}$ (of type A) where the different reactions dominate.

electron-electron bremsstrahlung dominates. Figure 6 differs from Fig. 5 mainly by the increase of the efficiency of neutrino emission due to Cooper pairing of neutrons (at $\left.\rho=2 \rho_{0}\right)$ and direct Urca process (at $\rho=5 \rho_{0}$ ).

It is well known that only one neutrino process dominates at a given density in a non-superfluid neutron-star core. It is either direct Urca or modified Urca process. The situation is drastically different in superfluid matter. As seen from Figs. 5 and 6, Cooper-pairing neutrino emission becomes dominant in the presence of a weak neutron superfluidity. With the increase of $T_{\mathrm{cn}}$ in the superfluid regime, modified Urca process becomes unimportant and Cooper-pairing neutrino emission dominates.

\section{Conclusions}

We have calculated the factors which describe the reduction of the neutrino emissivity in the neutron and proton branches of modified Urca process by superfluidities of neutrons and protons. We have considered singlet-state pairing of protons (pairing A) and either singlet-state or triplet-state pairing of neutrons (A, B or $\mathrm{C}$ ). The reduction factors are fitted by analytic expressions presented in Appendix to facilitate their use in computer codes.

We have also considered the reduction of neutrino bremsstrahlung due to neutron-neutron and neutronproton scattering by proton superfluidity A and neutron 
superfluidity C. We have constructed the approximate reduction factors and fitted them by analytic expressions. We have determined also the dominant neutrino emission mechanisms in a neutron star core at different values of the critical temperatures of the neutron and protons, $T_{\mathrm{cn}}$ and $T_{\mathrm{cp}}$, for the cases of neutron superfluidity of type $\mathrm{B}$ or $\mathrm{C}$.

Our results combined with those known in the literature (e.g., Yakovlev et al. 2001) allow one to calculate the neutrino emissivity in a neutron-star core in the presence of proton superfluidity A and neutron superfluidity A, B, or $\mathrm{C}$. The results can be useful to study thermal evolution of neutron stars, first of all, cooling of isolated neutron stars. Our cooling simulations based on the present results will be published elsewhere.

Acknowledgements. I am grateful to D. G. Yakovlev for discussions, to M. Ulanov, and K. P. Levenfish for technical assistance, and to anonymous referee for useful remarks. The work was supported partly by RFBR (grants Nos. 02-02-17668 and 00-07-90183).

\section{Appendix}

In Fig. 1 we plot four regions, I, II, III, and IV, in the $v_{1}-v_{2}$ plane where the fit expressions for the neutron and proton branches of modified Urca process are different. This selection of the regions is the same for any type of neutron superfluidity, A, B, or C.

We have calculated the reduction factors of the modified Urca process from Eq. (22) as described in Sect. 2. Introducing the polar coordinates $\left(v_{1}=v \sin \varphi, v_{2}=\right.$ $v \cos \varphi$ ), in regions I, II, and III we fit the numerical results by the expression

$R^{\mathrm{N}}=\exp \left(-\frac{A v^{2}}{\left(1+B v^{2}\right)^{C}}\right)$

while in region IV we use the fit of the form

$R^{\mathrm{N}}=C \exp (-A / B)$

In regions I, II, and III, the functions $A, B$, and $C$ depend on $\varphi$. In region IV, they depend on $v_{1}$ and $v_{2}$.

In the case of neutron and proton superfluidity A for neutron branch of modified Urca process we get the following fits.

In region I:

$$
\begin{aligned}
A & =p_{1}+p_{2} t^{2}+\frac{p_{4}}{1+p_{3} t}-p_{5} t \\
B & =p_{6}+p_{7} t^{2}+\frac{p_{9}}{1+p_{8} t}-p_{10} t \\
C & =p_{11}-\frac{p_{12}}{y\left(1+p_{13} z^{2}\right)^{3}} \\
t & =\cos ^{2}(\varphi), y=\sin ^{2}\left(\varphi+p_{15}\right), \\
z & =\cos ^{2}\left(\varphi+p_{14}\right) .
\end{aligned}
$$

Table 1. Fit coefficients $p_{i}$ for the reduction factor $R_{\mathrm{AA}}^{\mathrm{n}}$; powers of 10 are given in square brackets.

\begin{tabular}{rrrrl}
\hline \hline$i$ & \multicolumn{1}{c}{ I } & \multicolumn{1}{c}{ II } & \multicolumn{1}{c}{ III } & \multicolumn{1}{c}{ IV } \\
\hline 1 & 0.257798 & -9.495146 & -2.678004 & 0.268730 \\
2 & 0.003532 & -1.909172 & 64.33063 & 0.089294 \\
3 & 19.57034 & 0.820250 & -2.736549 & 0.002913 \\
4 & 0.036350 & 10.17103 & 0.093232 & $1.752838[-5]$ \\
5 & 0.173561 & 5.874262 & 0.380818 & $3.047384[-7]$ \\
6 & 0.039996 & 0.023332 & -0.015405 & 0.022415 \\
7 & 0.101014 & 0.003191 & -16.79340 & 0.001835 \\
8 & 16.61755 & 201.8576 & 112.4511 & $5.849410[-7]$ \\
9 & 0.063353 & 5.520899 & 517.5343 & 0.001610 \\
10 & 0.101188 & 1.257021 & 0.134529 & \\
11 & 0.343374 & -2.367854 & -0.174503 & \\
12 & -0.135307 & 1.096571 & -0.029008 & \\
13 & 2.404372 & 0.481874 & 1.277903 & \\
14 & 1.055914 & 487.4290 & -25.70616 & \\
15 & 1.086360 & -0.452688 & 558.1592 & \\
16 & & -257.9342 & 0.328108 & \\
17 & & 17.83708 & 0.642631 & \\
18 & & & 0.260288 & \\
\hline
\end{tabular}

In region II:

$A=p_{1}+p_{2} z^{2}+\frac{p_{4}}{1+p_{3} z}+p_{5} z$,

$B=p_{6}-\frac{p_{7} q}{1+p_{11} t^{2}}+p_{12} q t^{2}$,

$C=p_{13}+p_{14} y^{3}+\frac{p_{16} y^{3}}{1+p_{15} z^{2}}-p_{17} y^{2}$,

$z=\cos ^{2} \varphi, \quad t=\cos ^{2}\left(\varphi+p_{8}\right)$

$q=\sin ^{2}\left(p_{9} \varphi+p_{10}\right), \quad y=\sin ^{2}\left(\varphi+\frac{3 \pi}{4}\right)$.

In region III:

$A=p_{4}+\frac{p_{1} y}{1+p_{2} y+p_{3} y^{2}}+p_{5} y+p_{6} y t$,

$B=p_{10}+\frac{p_{7} y}{1+p_{8} y+p_{9} y^{2}}+p_{11} y+p_{12} y t$,

$C=p_{16}+\frac{p_{13} y}{1+p_{14} y+p_{15} y^{2}}+p_{17} y+p_{18} y t$,

$t=\sin ^{2}\left(\frac{2 \pi \varphi}{0.321750554}\right), \quad y=\sin ^{2} \varphi$.

In region IV:

$A=p_{1} v_{1}^{2}+p_{2} v_{2}^{2}+p_{3} v_{1}^{2} v_{2}^{2}+p_{4} v_{1}^{6}+p_{5} v_{2}^{6}$,

$B=1+p_{6} v_{1}^{2}+p_{7} v_{2}^{2}+p_{8} v_{1}^{4}$

$C=1+p_{9} v_{2}^{4}$.

Coefficients $p_{i}$ which enter Eqs. (A3)-(A6) are listed in Table 1 . The calculation and fit errors do not exceed $10 \%$ for $R_{\mathrm{AA}}^{\mathrm{n}} \gtrsim 10^{-5}$. 
Table 2. Coefficients $p_{i}$ for $R_{\mathrm{AB}}^{\mathrm{n}}$.

\begin{tabular}{rrrrl}
\hline \hline$i$ & \multicolumn{1}{c}{ I } & \multicolumn{1}{c}{ II } & \multicolumn{1}{c}{ III } & \multicolumn{1}{c}{ IV } \\
\hline 1 & -0.719681 & -6.475443 & 0.316041 & 0.565001 \\
2 & -0.024591 & -1.186294 & -289.2964 & 0.087929 \\
3 & 0.297357 & 0.591347 & 2480.961 & 0.006756 \\
4 & 1.260056 & 6.953996 & -268.8219 & $1.667194[-4]$ \\
5 & 0.100466 & 3.366945 & 1984.115 & $3.782805[-6]$ \\
6 & 0.148464 & -9.172994 & 3503.094 & 0.173165 \\
7 & 0.253881 & -2.675793 & 0.331551 & $1.769413[-5]$ \\
8 & 140.3699 & 1.053679 & -0.265977 & $7.710124[-8]$ \\
9 & 0.132615 & 10.38526 & 1098.324 & 0.001695 \\
10 & 0.280765 & 7.138369 & 65528.01 & \\
11 & 0.375796 & & 0.024500 & \\
12 & -0.096843 & & 0.120536 & \\
13 & 3.100942 & & 89.79866 & \\
14 & 0.275434 & & 5719.134 & \\
15 & 0.330574 & & 285.8473 & \\
16 & & 0.402111 & \\
17 & & & 16657.19 & \\
\hline
\end{tabular}

In the case of proton superfluidity A and neutron superfluidity B for neutron branch of modified Urca process we get the following fits. In region I:

$$
\begin{aligned}
A & =p_{1}+p_{2} t^{2}+\frac{p_{4}}{1+p_{3} t}-p_{5} t \\
B & =p_{6}+p_{7} t^{2}+\frac{p_{9} t}{1+p_{8} t^{2}}-p_{10} t \\
C & =p_{11}-\frac{p_{12}}{y\left(1+p_{13} z^{2}\right)^{3}} \\
y & =\sin ^{2}\left(\varphi+p_{15}\right), \quad t=\cos ^{2} \varphi \\
z & =\cos ^{2}\left(\varphi+p_{14}\right)
\end{aligned}
$$

In region II:

$$
\begin{aligned}
A & =p_{1}+p_{2} z^{2}+\frac{p_{4}}{1+p_{3} z}+p_{5} z, \\
B & =0.035, \\
C & =p_{6}+p_{7} z^{2}+\frac{p_{9}}{1+p_{8} z}+p_{10} z, \\
z & =\cos ^{2} \varphi, \quad t=\cos ^{2}\left(\varphi+p_{8}\right), \\
q & =\sin ^{2}\left(p_{9} \varphi+p_{10}\right), \quad y=\sin ^{2}(\varphi+3 \pi / 4) .
\end{aligned}
$$

In region III:

$$
\begin{aligned}
& A=\frac{p_{12}\left(1+p_{15} \varphi^{2}+p_{16} \varphi^{3}+p_{17} \varphi^{4}\right)}{1+p_{13} \varphi^{2}+p_{14} \varphi^{3}} \\
& B=p_{11}+\frac{p_{7}}{1+p_{8} \varphi^{2}+p_{9} \varphi^{3}+p_{10} \varphi^{4}} \\
& C=\frac{p_{1}\left(1+p_{4} \varphi^{2}+p_{5} \varphi^{3}+p_{6} \varphi^{4}\right)}{1+p_{2} \varphi^{2}+p_{3} \varphi^{3}}
\end{aligned}
$$

In region IV:

$$
\begin{aligned}
& A=p_{1} v_{1}^{2}+p_{2} v_{2}^{2}+p_{3} v_{1}^{2} v_{2}^{2}+p_{4} v_{1}^{6}, \\
& B=\sqrt{1+p_{6} v_{1}^{2}+p_{7} v_{2}^{2}+p_{5} v_{1}^{8}+p_{8} v_{1}^{6}}, \\
& C=1+p_{9} v_{2}^{4} .
\end{aligned}
$$

Table 3. Coefficients $p_{i}$ for $R_{\mathrm{AB}}^{\mathrm{p}}$.

\begin{tabular}{rrrrl}
\hline \hline$i$ & \multicolumn{1}{c}{ I } & \multicolumn{1}{c}{ II } & \multicolumn{1}{c}{ III } & \multicolumn{1}{c}{ IV } \\
\hline 1 & 0.288203 & 0.398261 & 0.387542 & 0.272730 \\
2 & -0.124974 & -0.054952 & -195.5462 & 0.165858 \\
3 & 17.39273 & -0.084964 & 3032.985 & 0.005903 \\
4 & 0.083392 & -0.036240 & -189.0452 & $2.555386[-5]$ \\
5 & 0.059046 & -0.168712 & 3052.617 & $2.593057[-7]$ \\
6 & 0.028084 & -0.704750 & 442.6031 & 0.023930 \\
7 & -0.019990 & -0.066981 & 0.041901 & 0.006180 \\
8 & 28.37210 & 1.223731 & -0.022201 & $1.289532[-5]$ \\
9 & 0.244471 & 0.363094 & 5608.168 & 0.005368 \\
10 & -0.610470 & -0.357641 & -10761.76 & \\
11 & 0.023288 & 0.869196 & 0.064643 & \\
12 & 0.475196 & -0.364248 & 0.296253 & \\
13 & -0.180420 & 2.668230 & 106.3387 & \\
14 & 25.51325 & -0.765093 & -75.36126 & \\
15 & 0.281721 & -4.198753 & 84.65801 & \\
16 & -0.080480 & & 0.530223 & \\
17 & -0.191637 & & -86.76801 & \\
\hline
\end{tabular}

Coefficients $p_{i}$ which enter Eqs. (A7)-(A10) are listed in Table 2. The calculation and fit errors do not exceed 5$15 \%$ in those cases in which $R_{\mathrm{AB}}^{\mathrm{n}} \gtrsim 10^{-5}$.

In the case of proton superfluidity $\mathrm{A}$ and neutron superfluidity B for proton branch of modified Urca process we get the following fits.

In region $\mathrm{I}$ :

$$
\begin{aligned}
& A=p_{1}+p_{2} \varphi+\frac{p_{4} \varphi}{\left(1+p_{3} t \varphi\right)^{2}}+p_{5} t \varphi^{2}, \\
& B=p_{6}+p_{7} \varphi+\frac{p_{9} \varphi}{\left(1+p_{8} t \varphi+p_{10} y t\right)^{2}}+p_{11} \varphi^{2}, \\
& C=p_{12}-p_{13} t+\frac{p_{16}}{\left(1+p_{14} t^{2}\right)^{2}}+p_{17} t \varphi, \\
& y=\sin ^{2}\left(\varphi+p_{15}\right), \quad t=\cos ^{2} \varphi .
\end{aligned}
$$

In region II:

$$
\begin{aligned}
A & =p_{1}+p_{2} z^{2}+\frac{p_{4}}{1+p_{3} z}+p_{5} \varphi \\
B & =p_{6}+p_{7} z^{2}+\frac{p_{8}}{1+p_{9} z}+p_{10} \varphi \\
C & =p_{11}-p_{12} \varphi^{2}+\frac{p_{13} \varphi^{2}}{1+p_{14} z}+p_{15} \varphi \\
z & =\cos ^{2} \varphi .
\end{aligned}
$$

In region III the factors $A, B$, and $C$ have the same form as in Eq. (A9).

In region IV:

$A=p_{1} v_{2}^{2}+p_{2} v_{1}^{2}+p_{3} v_{1}^{2} v_{2}^{2}+p_{4} v_{2}^{6}+p_{5} v_{1}^{6}$,

$B=1+p_{6} v_{2}^{2}+p_{7} v_{1}^{2}+p_{8} v_{2}^{4}$,

$C=1+p_{9} v_{1}^{4}$.

Coefficients $p_{i}$ in (A9), (A11)-(A13) are given in Table 3. Calculation and fit errors do not exceed $8 \%$ for those values of $v_{1}$ and $v_{2}$ for which $R_{\mathrm{AB}}^{\mathrm{p}} \gtrsim 10^{-5}$. 
Table 4. Coefficients $p_{i}$ for $R_{\mathrm{AC}}^{\mathrm{n}}$.

\begin{tabular}{rrrrl}
\hline \hline$i$ & \multicolumn{1}{c}{$\mathrm{I}$} & \multicolumn{1}{c}{ II } & \multicolumn{1}{c}{ III } & \multicolumn{1}{c}{ IV } \\
\hline 1 & 0.897393 & -3.471368 & 0.322115 & 0.175090 \\
2 & -0.045357 & -0.133540 & -15.05047 & 0.088159 \\
3 & 0.309724 & 0.143230 & 112.9733 & $3.055763[-3]$ \\
4 & -0.739962 & 3.634659 & -13.79012 & $3.984607[-7]$ \\
5 & 0.222597 & 0.496579 & 128.3156 & $5.591497[-8]$ \\
6 & 0.032104 & 0.030609 & 39.82789 & 0.046496 \\
7 & -0.054011 & 0.005056 & 0.164614 & $1.452790[-5]$ \\
8 & 61.73448 & 0.438608 & 49.07699 & $4.505614[-8]$ \\
9 & 0.195679 & -2.970431 & -3.145006 & $1.779724[-3]$ \\
10 & -0.001851 & 0.284703 & 5132.076 & $2.136809[-4]$ \\
11 & 0.482581 & 0.898355 & 0.018737 & $5.365717[-4]$ \\
12 & -0.001637 & -0.036420 & 0.100223 & \\
13 & -0.685659 & 0.407393 & 4.055407 & \\
14 & 1.528415 & -0.058942 & 390.6242 & \\
15 & -0.053834 & 0.605413 & 6.594365 & \\
16 & -0.452426 & 2.851209 & 175.7396 & \\
17 & -0.053502 & -0.800218 & 441.3965 & \\
18 & & 1.497718 & & \\
19 & & 1.476375 & & \\
\hline
\end{tabular}

In the case of proton superfluidity $\mathrm{A}$ and neutron superfluidity $\mathrm{C}$ for neutron branch of modified Urca process we get the following fits.

In region $\mathrm{I}$ :

$A=p_{1}+p_{2} t^{2}+\frac{p_{4}}{1+p_{3} t}-p_{5} t$

$B=p_{6}+p_{7} t^{2}+\frac{p_{9} t}{\left(1+p_{8} t q\right)^{2}}-p_{10} t$

$C=p_{11}-\frac{p_{12}}{s\left(1+p_{13} z^{2}\right)^{3}}+p_{17} s^{2}$,

$t=\cos ^{2} \varphi, \quad z=\cos ^{2}\left(\varphi+p_{14}\right)$,

$q=\sin ^{2}\left(\varphi+p_{16}\right), \quad s=\sin ^{2}\left(\varphi+p_{15}\right)$.

(A14)

In region II:

$A=p_{1}+p_{2} z^{2}+\frac{p_{4}}{1+p_{3} z}+p_{5} z$,

$B=p_{6}-\frac{p_{7} q_{\mathrm{b}}}{1+p_{11} t_{\mathrm{b}}^{2}}+p_{12} q_{\mathrm{b}} t_{\mathrm{b}}^{2}$,

$C=p_{13}-\frac{p_{14} q_{\mathrm{c}}}{1+p_{18} t_{\mathrm{c}}^{2}}+p_{19} q_{\mathrm{c}} t_{\mathrm{c}}^{2}$,

$z=\cos ^{2} \varphi, \quad t_{\mathrm{b}}=\cos ^{2}\left(\varphi+p_{8}\right)$,

$q_{\mathrm{b}}=\sin ^{2}\left(p_{9} \varphi+p_{10}\right), \quad t_{\mathrm{c}}=\cos ^{2}\left(\varphi+p_{15}\right)$,

$q_{\mathrm{c}}=\sin ^{2}\left(p_{16} \varphi+p_{17}\right)$.

(A15)

In region III the factors $A, B$, and $C$ have the same form as in Eq. (A9). In region IV:

$$
\begin{aligned}
& A=p_{1} v_{1}^{2}+p_{2} v_{2}^{2}+p_{3} v_{1}^{2} v_{2}^{2}+p_{4} v_{1}^{6}, \\
& B=\sqrt{1+p_{6} v_{1}^{2}+p_{7} v_{2}^{2}+p_{5} v_{1}^{8}+p_{8} v_{1}^{6}}, \\
& C=1+p_{9} v_{2}^{4}+p_{10} v_{2}^{2}+p_{11} v_{1}^{2} v_{2}^{2} .
\end{aligned}
$$

Table 5. Coefficients $p_{i}$ for $R_{\mathrm{AC}}^{\mathrm{p}}$.

\begin{tabular}{rrrrl}
\hline \hline$i$ & \multicolumn{1}{c}{ I } & \multicolumn{1}{c}{ II } & \multicolumn{1}{c}{ III } & \multicolumn{1}{c}{ IV } \\
\hline 1 & 0.049947 & -4.985248 & 0.100241 & 0.272905 \\
2 & -0.029006 & -0.025984 & 0.005432 & 0.058684 \\
3 & 3872.363 & -0.007404 & -0.748377 & $2.053694[-3]$ \\
4 & 0.250385 & 5.294455 & 0.050631 & $1.800867[-7]$ \\
5 & -0.245758 & -0.201654 & 0.007900 & $1.911708[-8]$ \\
6 & 0.018241 & 0.184431 & -0.032915 & 0.052786 \\
7 & 0.090256 & -0.139729 & -0.000768 & $2.043824[-5]$ \\
8 & 108.8302 & 0.415562 & 0.044312 & $4.458912[-8]$ \\
9 & 1.007326 & 2.692073 & -0.697892 & $1.101541[-3]$ \\
10 & 0.061586 & -0.385832 & 0.032534 & $3.312811[-4]$ \\
11 & 0.797695 & 1.055347 & 0.080109 & $2.682799[-4]$ \\
12 & 175.5965 & 0.013667 & 0.031994 & \\
13 & 9.306619 & -0.509106 & 8.724039 & \\
14 & -0.551550 & -0.267675 & 2.982355 & \\
15 & 1.203014 & 0.034585 & -0.062076 & \\
16 & 0.096598 & & & \\
17 & -0.441039 & & & \\
\hline
\end{tabular}

Coefficients $p_{i}$ in (A14)-(A16) are given in Table 4. Calculation and fit errors do not exceed $10 \%$ for those values of $v_{1}$ and $v_{2}$ for which $R_{\mathrm{AC}}^{\mathrm{n}} \gtrsim 10^{-6}$.

In the case of proton superfluidity $\mathrm{A}$ and neutron superfluidity $\mathrm{C}$ for the proton branch of modified Urca process at $v_{1} \leq 25$ we get the following fits

In region $\mathrm{I}$ :

$$
\begin{aligned}
A & =p_{1}+p_{2} t^{2}+\frac{p_{4} t}{1+p_{3} t^{2}}-p_{5} t, \\
B & =p_{6}+p_{7} t q+\frac{p_{9} t}{\left(1+p_{8} t q\right)^{2}}-p_{10} t, \\
C & =p_{11}-\frac{p_{12} t}{s\left(1+p_{13} z^{2}\right)^{3}}+p_{17} s t, \\
q & =\sin ^{2}\left(\varphi+p_{15}\right), \quad s=\sin ^{2}\left(\varphi+p_{16}\right), \\
t & =\cos ^{2} \varphi, \quad z=\cos ^{2}\left(\varphi+p_{14}\right) .
\end{aligned}
$$

In region II:

$$
\begin{aligned}
A & =p_{1}+p_{2} z^{2}+\frac{p_{4}}{1+p_{3} z}+p_{5} \varphi \\
B & =p_{6}+p_{7} z^{2}+\frac{p_{8}}{1+p_{9} z}+p_{10} \varphi \\
C & =p_{11}+p_{12} z^{2}+\frac{p_{13}}{1+p_{14} z}+p_{15} \varphi \\
z & =\cos ^{2} \varphi
\end{aligned}
$$

In region III:

$A=p_{1}+p_{2} \varphi z+\frac{p_{4}}{1+p_{3} z}+p_{5} \varphi$,

$B=p_{6}+p_{7} \varphi t+\frac{p_{8} z}{1+p_{9} z^{2}}+p_{10} \varphi$,

$C=p_{11}+p_{12} \varphi z+\frac{p_{14}}{1+p_{13} z}+p_{15} \varphi$,

$t=\sin ^{2}\left(\frac{2 \pi \varphi}{0.321750554}\right), \quad z=\cos ^{2} \varphi$. 
Table 6. Maximum values of $v=v_{\max }$ in the fit expressions and maximum relative error $\delta_{\max }$ of the fits at $v \leq v_{\max }$.

\begin{tabular}{crrr}
\hline \hline Process & I & II & III \\
\hline$R_{\mathrm{AA}}^{\mathrm{n}}, v_{\max }$ & 20 & 23 & 25 \\
$\delta_{\max }, \%$ & $<10$ & $<15$ & $<20$ \\
$R_{\mathrm{AB}}^{\mathrm{n}}, v_{\max }$ & 15 & 15 & 25 \\
$\delta_{\max }, \%$ & $<20$ & $<20$ & $<50$ \\
$R_{\mathrm{AC}}^{\mathrm{n}}, v_{\max }$ & 19 & 21 & 26 \\
$\delta_{\max }, \%$ & $<15$ & $<26$ & $<20$ \\
$R_{\mathrm{AB}}^{\mathrm{p}}, v_{\max }$ & 22 & 13 & 13 \\
$\delta_{\max }, \%$ & $<20$ & $<5$ & $<3$ \\
$R_{\mathrm{AC}}^{\mathrm{p}}, v_{\max }$ & 23 & 16 & 13 \\
$\delta_{\max }, \%$ & $<13$ & $<5$ & $<2$ \\
\hline
\end{tabular}

In region IV:

$$
\begin{aligned}
& A=p_{1} v_{2}^{2}+p_{2} v_{1}^{2}+p_{3} v_{2}^{2} v_{1}^{2}+p_{4} v_{2}^{6}, \\
& B=\sqrt{1+p_{6} v_{2}^{2}+p_{7} v_{1}^{2}+p_{5} v_{2}^{8}+p_{8} v_{2}^{6},} \\
& C=1+p_{9} v_{1}^{4}+p_{10} v_{1}^{2}+p_{11} v_{2}^{2} v_{1}^{2} .
\end{aligned}
$$

Coefficients $p_{i}$ in (A17)-(A20) are given in Table 5 . Calculation and fit errors do not exceed $5 \%$ for those values for which $R_{\mathrm{AC}}^{\mathrm{p}} \gtrsim 10^{-6}$.

In Table 6 we give maximum values of $v=v_{\max }$ of our fit expressions in regions I, II, and III, and maximum fit errors $\delta_{\max }$ at $v \leq v_{\max }$ in these regions. These maximum errors occur at $v \sim v_{\max }$, where the reduction factors are very small (and are thus unimportant for calculation of the total neutrino emissivity). At $v>v_{\max }$ our fit expressions are not reliable and we recommend to set the corresponding reduction factors equal to zero in computer codes.

\section{References}

Amundsen, L., \& Østgaard, E. 1985, Nucl. Phys. A, 442, 163 Bahcall, J. N., \& Wolf, R. A. 1965, Phys. Rev., 140, 1452

Flowers, E. G., Ruderman, M., \& Sutherland, P. G. 1976, ApJ, 205,541

Friman, B. L., \& Maxwell, D. V. 1979, ApJ, 232, 541

Kaminker, A. D., \& Haensel, P. 1999, Acta Phys. Polonica, 30, 1125 [astro-ph/9908249]

Lattimer, J. M., Pethick, C. J., Prakash, M., \& Haensel, P. 1991, Phys. Rev. Lett., 66, 2701

Levenfish, K. P., \& Yakovlev, D. G. 1994, Astron. Lett., 20, 43

Levenfish, K. P., \& Yakovlev, D. G. 1996, Astron. Lett., 22, 47

Lifshitz, E. M., \& Pitaevskii, L. P. 1980, Statistical Physics, Part 2 (Pergamon, Oxford)

Lombardo, U., \& Schulze, H.-J. 2001, in Physics of Neutron Star Interiors, ed. D. Blaschke, N. Glendenning, \& A. Sedrakian (Springer, Berlin), 30

Musikar, P., Sauls, J. A., \& Serene, J. W. 1980, Phys. Rev. D, 21,1494

Page, D., \& Applegate, J. H. 1992, ApJ, 394, L17

Prakash, M., Ainsworth, T. L., \& Lattimer, J. M. 1988, Phys. Rev. Lett., 61, 2518

Shapiro, S. L., \& Teukolsky, S. A. 1983. Black Holes, White Dwarfs, and Neutron Stars (Wiley-Interscience, New York)

Tamagaki, R. 1971, Prog. Theor. Phys., 44, 905

Yakovlev, D. G., Kaminker, A. D., Gnedin, O. Y., \& Haensel, P. 2001, Phys. Rep., 354, 1

Yakovlev, D. G., \& Levenfish, K. P. 1995, A\&A, 297, 717

Yakovlev, D. G., Kaminker, A. D., \& Levenfish, K. P. 1999, A\&A, 343650

Yakovlev, D. G., Levenfish, K. P., \& Shibanov, Yu. A. 1999, Physics - Uspekhi 42, 737 [astro-ph/9906456] 